\title{
Simulation of Turbulent Flow Inside and Above Wind Farms: Model Validation and Layout Effects
}

\author{
Yu-Ting Wu • Fernando Porté-Agel
}

Received: 24 February 2012 / Accepted: 2 July 2012 / Published online: 25 July 2012

(C) Springer Science+Business Media B.V. 2012

\begin{abstract}
A recently-developed large-eddy simulation framework is validated and used to investigate turbulent flow within and above wind farms under neutral conditions. Two different layouts are considered, consisting of thirty wind turbines occupying the same total area and arranged in aligned and staggered configurations, respectively. The subgrid-scale (SGS) turbulent stress is parametrized using a tuning-free Lagrangian scale-dependent dynamic SGS model. The turbine-induced forces are modelled using two types of actuator-disk models: (a) the 'standard' actuator-disk model (ADM-NR), which calculates only the thrust force based on one-dimensional momentum theory and distributes it uniformly over the rotor area; and (b) the actuator-disk model with rotation (ADM-R), which uses blade-element momentum theory to calculate the lift and drag forces (that produce both thrust and rotation), and distributes them over the rotor disk based on the local blade and flow characteristics. Validation is performed by comparing simulation results with turbulence measurements collected with hot-wire anemometry inside and above an aligned model wind farm placed in a boundarylayer wind tunnel. In general, the ADM-R model yields improved predictions compared with the ADM-NR in the wakes of all the wind turbines, where including turbine-induced flow rotation and accounting for the non-uniformity of the turbine-induced forces in the ADM-R appear to be important. Another advantage of the ADM-R model is that, unlike the ADM$\mathrm{NR}$, it does not require a priori specification of the thrust coefficient (which varies within a wind farm). Finally, comparison of simulations of flow through both aligned and staggered wind farms shows important effects of farm layout on the flow structure and wind-turbine performance. For the limited-size wind farms considered in this study, the lateral interaction between cumulated wakes is stronger in the staggered case, which results in a farm wake that is more homogeneous in the spanwise direction, thus resembling more an internal boundary layer. Inside the staggered farm, the relatively longer separation between consecutive downwind turbines allows the wakes to recover more, exposing the turbines to higher local wind speeds (leading to higher turbine efficiency) and lower turbulence intensity levels (leading to
\end{abstract}

Y.-T. Wu · F. Porté-Agel $(\bowtie)$

Wind Engineering and Renewable Energy Laboratory (WIRE), École Polytechnique Fédérale de Lausanne (EPFL), EPFL-ENAC-IIE-WIRE, 1015 Lausanne, Switzerland

e-mail: fernando.porte-agel@epfl.ch 
lower fatigue loads), compared with the aligned farm. Above the wind farms, the area-averaged velocity profile is found to be logarithmic, with an effective wind-farm aerodynamic roughness that is larger for the staggered case.

Keywords Actuator-disk model $\cdot$ Blade-element momentum theory $\cdot$ Effective roughness · Large-eddy simulation · Wind-farm wakes

\section{Introduction}

Driven by the worldwide growing demand for green energy, many large wind farms have been built and others are being planned in both offshore and onshore areas with abundant wind resources. Due to the clustering of many wind turbines on finite surface areas, the effect of multiple wakes from upwind turbines leads to a significant reduction in power production as well as to an increase in long-term dynamic fatigue loads inside wind farms (Vermeer et al. 2003; Hansen et al. 2006; Sørensen 2011). For example, in the well-known Horns Rev offshore wind farm, the reduction of power inside the farm (with respect to the first row of turbines) is found to be as large as 25 to $45 \%$, depending on the wind direction (Barthelmie et al. 2007, 2009; Hansen et al. 2012). Such large power losses depend on the distribution of the wind velocity inside the wind farm, which is modulated by the complex interactions between the turbulent atmospheric boundary-layer (ABL) flow and the multiple wind-turbine wakes. This ABL-wind-farm interaction is in turn affected by several factors such as atmospheric stability, land-surface characteristics, wind-farm layout and wind direction. Accurate numerical prediction of the ABL flow and its interaction with wind turbines and wind farms is needed for optimizing the design of wind energy projects (wind-turbine siting) and their operation. Particularly, it can be used to maximize power output and minimize fatigue loading on downwind turbines. In addition, numerical simulations can provide valuable quantitative insights into the potential impacts of wind farms on local boundary-layer meteorology.

Various numerical studies have investigated fundamental features of axisymmetric wakes behind stand-alone turbines placed in laminar free-stream flows by solving the unsteady Euler or Navier-Stokes equations, and modelling the turbine effects with actuator-disk and actuator-line models (Sørensen and Myken 1992; Sørensen and Kock 1995; Masson et al. 1997; Sørensen and Shen 2002). In spite of the important insights obtained from the study of axisymmetric wakes in laminar flows, there is a need for simulations of wind-turbine wakes that consider the effects of more realistic turbulent boundary-layer flow conditions (including wind shear, turbulence and atmospheric stability effects). Previous studies of ABL flows through isolated wind turbines have modelled the turbulent flow using the Reynolds-averaged Navier-Stokes (RANS) approach (e.g., Alinot and Masson 2002; Gómez-Elvira et al. 2005; Kasmi and Masson 2008) and, more recently, the large-eddy simulation (LES) technique (e.g., Jimenez et al. 2007, 2008, 2010; Wu and Porté-Agel 2011). Note that RANS solves the time-averaged Navier-Stokes equations and, therefore, the effect of all the turbulence scales on the turbulent fluxes has to be parametrized. In contrast, LES explicitly resolves all scales of turbulent transport larger than the filter scale (of the same size or slightly larger than the grid size), while the effect of the smallest scales is parametrized using a subgrid-scale (SGS) model. Due to the fact that a large fraction of the turbulence is resolved, LES can be considered as the state-of-the-art numerical technique for the simulation of turbulent flows in general, and the ABL in particular. For wind energy applications, besides a SGS turbulence model, LES requires also a parametrization for the effect of the wind turbines on the flow. This can be done using actuator-disk models or actuator-line models. 
Both RANS and LES techniques have also been recently used for the simulation of ABL flow through wind farms. In the context of RANS, Ammara et al. (2002) employed the actuator-disk approximation in combination with the blade-element momentum (BEM) theory to investigate the interaction of multiple wakes for different incoming wind directions. Calaf et al. (2010) used LES with a standard actuator-disk model, which considers a uniform thrust load over the rotor disk in the Betz limit condition, and investigated the effective aerodynamic roughness and the friction velocity above an infinite wind farm. Lu and Porté-Agel (2011) employed the actuator-line model in the context of LES to study the effects of very large wind farms on the flow structure and the turbulent fluxes of momentum and heat in a stably-stratified ABL. Porté-Agel et al. (2011) used LES with actuator-disk and actuatorline models to simulate the ABL flow through an operational wind farm, where both sodar wind measurements and turbine power data were available for model validation. Despite the various efforts to simulate turbulent flow in wind farms, to the best of our knowledge, no rigorous validation study has been performed to compare systematically simulated results against high-resolution measurements of turbine wakes inside and above a wind farm. Moreover, there is still limited understanding of how the turbine wakes affect the performance of downwind turbines as well as the flow and turbulent transport of momentum in large wind farms with different configurations.

In this study, we validate a recently-developed LES framework in the simulation of a neutrally-stratified turbulent boundary-layer flow through an aligned wind farm. In this framework, the SGS stresses and the turbine-induced forces are parametrized using a tuning-free Lagrangian scale-dependent dynamic model (Stoll and Porté-Agel 2006) and two types of actuator-disk models (Wu and Porté-Agel 2011), respectively. The framework and model formulations are presented in Sect. 2, and the LES technique is then tested against windtunnel measurements of turbulence characteristics collected in the wakes of miniature wind turbines deployed in an aligned wind farm (Chamorro and Porté-Agel 2011). The numerical and wind-tunnel experiments are described in Sect. 3. The results for model validation, as well as a comparison of the simulation results for the aligned and staggered wind-farm cases, are presented in Sect. 4, and a summary is given in Sect. 5 .

\section{LES Framework}

\subsection{LES Governing Equations and SGS Modelling}

LES solves the filtered incompressible Navier-Stokes equations, which can be written in rotational form as:

$$
\begin{aligned}
\frac{\partial \widetilde{u}_{i}}{\partial x_{i}} & =0 \\
\frac{\partial \widetilde{u}_{i}}{\partial t}+\widetilde{u}_{j}\left(\frac{\partial \widetilde{u}_{i}}{\partial x_{j}}-\frac{\partial \widetilde{u}_{j}}{\partial x_{i}}\right) & =-\frac{1}{\rho} \frac{\partial \widetilde{p}^{*}}{\partial x_{i}}-\frac{\partial \tau_{i j}^{d}}{\partial x_{j}}+v \frac{\partial^{2} \widetilde{u}_{i}}{\partial x_{j}^{2}}-\frac{f_{i}}{\rho}+\mathcal{F}_{i},
\end{aligned}
$$

where the tilde represents a three-dimensional spatial filtering operation at scale $\Delta, \tilde{u}_{i}$ is the resolved velocity in the $i$-th direction (with $i=1,2,3$ corresponding to the streamwise $(x)$, spanwise $(y)$ and vertical $(z)$ directions), $\rho$ is the air density, $v$ is the kinematic viscosity of air, $f_{i}$ is an immersed force (per unit volume) for modelling the effect of wind turbines on the flow, and $\mathcal{F}_{i}$ is a forcing term (e.g., a mean pressure gradient). The modified pressure $\widetilde{p}^{*}$ is defined as 


$$
\widetilde{p}^{*}=\widetilde{p}+\frac{1}{2} \rho \widetilde{u}_{k} \widetilde{u}_{k}+\frac{1}{3} \rho\left(\widetilde{u_{k} u_{k}}-\widetilde{u}_{k} \widetilde{u}_{k}\right)
$$

where $\widetilde{p}$ is the pressure, and the repeated subscript $k$ denotes summation. Also, $\tau_{i j}^{d}$ is the deviatoric part of the SGS stress and is modelled using a Smagorinsky eddy-viscosity model (Smagorinsky 1963),

$$
\tau_{i j}^{d}=-2\left(C_{\mathrm{S}} \Delta\right)^{2}|\widetilde{S}| \widetilde{S}_{i j}
$$

where $\widetilde{S}_{i j}=\left(\partial \widetilde{u}_{i} / \partial x_{j}+\partial \widetilde{u}_{j} / \partial x_{i}\right) / 2$ is the resolved strain rate tensor, $|\widetilde{S}|=\sqrt{2 \widetilde{S}_{i j} \widetilde{S}_{i j}}$ is the strain rate magnitude, and $C_{\mathrm{S}}$ is the Smagorinsky model coefficient.

One of the main challenges in the implementation of the Smagorinsky model is the specification of the model coefficient. Evidence from both numerical simulations (Porté-Agel et al. 2001a; Wan et al. 2007) and experimental a priori studies (Porté-Agel et al. 2001b; Kleissl et al. 2003) showed that the value of the model coefficient should decrease in regions of the flow with large local mean shear. To account for the spatial and temporal variation of the model coefficient in complex wind-farm wake flows, here we adopt the Lagrangian scaledependent dynamic model (Stoll and Porté-Agel 2006) to compute dynamically (without any ad hoc tuning) the optimized local value of the model coefficient $C_{\mathrm{S}}$ based on the dynamics of the resolved flow field. In the model, $C_{\mathrm{S}}^{2}$ at the grid scale $\Delta$ (Stoll and Porté-Agel 2006) can be calculated by

$$
C_{\mathrm{S}}^{2}(\Delta)=\frac{\left\langle L_{i j} M_{i j}\right\rangle_{\mathcal{L}}}{\left\langle M_{i j} M_{i j}\right\rangle_{\mathcal{L}}},
$$

where $\langle\cdot\rangle_{\mathcal{L}}$ denotes Lagrangian averaging, $L_{i j}=\overline{\widetilde{u}}_{i} \widetilde{u}_{j}-\overline{\widetilde{u}}_{i} \overline{\widetilde{u}}_{j}$ is the so-called Leonard stress tensor, $M_{i j}=2 \Delta^{2}\left(|\widetilde{\widetilde{S}}| \widetilde{S}_{i j}-\alpha^{2} \beta|\widetilde{\widetilde{S}}| \widetilde{\widetilde{S}}_{i j}\right)$, the overbar implies filtering at a test-filter scale $\bar{\Delta}=\alpha \Delta$ (with $\alpha>1$, typically $\alpha=2$ ), and $\beta=C_{\mathrm{S}}^{2}(\bar{\Delta}) / C_{\mathrm{S}}^{2}(\Delta)$ is a scale-dependence parameter, which is also determined dynamically in the simulation. More details on the formulation of scale-dependent dynamic models for the SGS stress and the SGS scalar fluxes can be found in Porté-Agel et al. (2000), Porté-Agel (2004), Stoll and Porté-Agel (2006), and Stoll and Porté-Agel (2008). The model has been successfully validated in simulations of ABL flow over both flat and complex terrain, under different thermal stratification conditions (PortéAgel et al. 2000; Stoll and Porté-Agel 2006; Wan et al. 2007; Wan and Porté-Agel 2011). Recently, Wu and Porté-Agel (2011) used this model to simulate a neutral boundary-layer flow through a stand-alone wind turbine. They reported values of the dynamically-computed coefficient that are smaller near the surface and on the edge of the turbine wake, where the shear is stronger.

\subsection{Wind-Turbine Parametrizations}

Using an actuator-disk approximation is a common approach to parametrize the turbineinduced forces (e.g., thrust, lift, and drag) in numerical models of flow through propellers (e.g., Phillips et al. 2009) and turbines (e.g., Ammara et al. 2002). This approach assumes the flow surrounding a wind turbine to be inviscid and does not require resolving the boundarylayer flow around the surface of the turbine, which decreases greatly the computational cost. Betz (1926) first applied a Rankine-Froude actuator disk method to determine the thrust force $(T)$ and the power production $(P)$ on an ideal turbine rotor and, thus, derived the well-known Betz limit for the maximum achievable efficiency of a wind turbine (maximum power coefficient of $C_{\mathrm{P}, \max }=16 / 27$ ). Due to its simplicity and capability to deliver reasonable results 


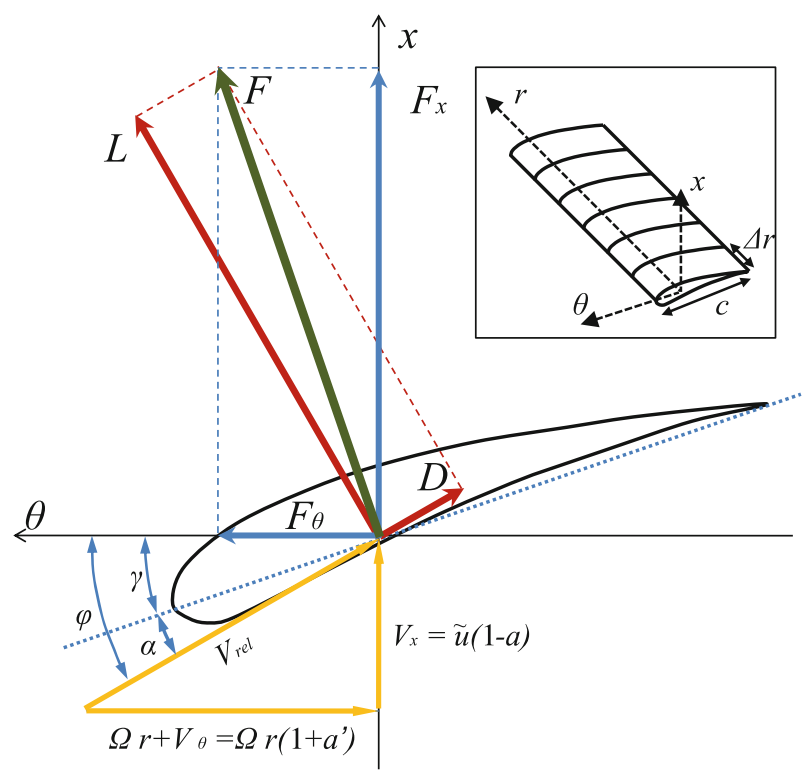

Fig. 1 A cross-sectional aerofoil element

with coarse grids, this method has been widely used in numerical simulations of turbines in the context of both RANS (Gómez-Elvira et al. 2005) and, more recently, LES (Calaf et al. 2010; Wu and Porté-Agel 2011; Porté-Agel et al. 2011). Since this method only considers a uniform thrust load over the rotor disk based on one-dimensional momentum theory (i.e., the rotational effect is not considered), here we refer to this method as the actuator-disk model without rotation (ADM-NR). In the ADM-NR, the thrust force per unit volume is modelled as $f_{x}^{\text {disk }}=\frac{1}{2} \frac{\rho}{\Delta x} \tilde{u}_{0}^{2} C_{\mathrm{T}}$, where $\tilde{u}_{0}$ is the unperturbed resolved streamwise velocity of the incident flow in the centre of the rotor disk, $C_{\mathrm{T}}$ is the thrust coefficient, and $\Delta_{x}$ is the size of a grid cell in the streamwise direction.

A major advancement in wind-turbine modelling was the introduction of the BEM theory. This theory considers that each blade of a wind turbine can be divided into $N$ blade elements (see Fig. 1), and which are assumed to behave aerodynamically as two-dimensional aerofoils and to have no radial action on the flow. Based on momentum balance around the aerofoils, the aerodynamic forces are determined using the lift and drag characteristics of the aerofoil as well as the local flow conditions. Note that, for each blade element, the lift and drag forces are perpendicular and parallel, respectively, to the direction of the local relative velocity. The resultant force is non-uniformly distributed over the rotor and produces thrust as well as rotation of the flow. Here, this approach will be referred to as the actuator-disk model with rotation (ADM-R). Figure 1 shows a schematic of the various forces, velocities and angles for a cross-sectional blade element at radius $r$ in the $(x, \theta)$ plane of the cylindrical coordinate system, looking down from the blade tip. The axial and tangential velocities of the incident flow at the blades are denoted as $V_{x}=V_{x}(r, \theta)$ and $V_{\theta}=V_{\theta}(r, \theta)$, respectively, in the inertial frame of reference. The local relative velocity with respect to the aerofoil element is defined as $\mathbf{V}_{\text {rel }}=\left(V_{x}, \Omega r+V_{\theta}\right)$, where $\Omega$ is the turbine angular velocity. The angle of attack is defined as $\alpha=\phi-\gamma$, where $\phi=\tan ^{-1}\left[V_{x} /\left(\Omega r+V_{\theta}\right)\right]$ is the angle between $V_{\text {rel }}$ and the rotor plane and $\gamma$ is the local pitch angle. In the ADM-R model, the resulting force per unit volume is given by 


$$
\mathbf{f}^{\text {disk }}=\frac{\rho V_{\mathrm{rel}}^{2}}{2 \Delta_{x}} \frac{B c}{2 \pi r}\left(C_{\mathrm{L}} \mathbf{e}_{\mathrm{L}}+C_{\mathrm{D}} \mathbf{e}_{\mathrm{D}}\right),
$$

where $C_{\mathrm{L}}=C_{\mathrm{L}}\left(\alpha, R e_{\mathrm{c}}\right)$ and $C_{\mathrm{D}}=C_{\mathrm{D}}\left(\alpha, R e_{\mathrm{c}}\right)$ are the lift and drag coefficients obtained from tabulated aerofoil data, respectively, $R e_{\mathrm{c}}$ is the Reynolds number based on the relative velocity and chord length, $\mathbf{e}_{\mathrm{L}}$ and $\mathbf{e}_{\mathrm{D}}$ are the directions of the unit vector for the lift and drag forces, respectively, $\Delta r$ is the radial length of the two-dimensional aerofoil element, $c$ is the chord length, and $B$ is the number of blades.

One important issue is how the thrust coefficient used in the ADM-NR model is specified a priori in simulations of flow through wind farms. This is due to the fact that the wind turbines should have different thrust coefficients due to their individual pitch control and the differences in the incoming flow due to the cumulative wake effects. To avoid this issue, in this study the thrust coefficient $C_{\mathrm{T}}$ is estimated based on the thrust force obtained from the $\operatorname{drag}(D)$ and lift $(L)$ forces computed using BEM theory in the ADM-R. Since the ADM-NR model considers an overall thrust force $(T)$ and ignores the effect of the turbine-induced flow rotation, a constant thrust coefficient applied on the whole rotor disk is calculated using

$$
C_{\mathrm{T}}=\frac{T}{0.5 \rho \tilde{u}_{0}^{2} A_{\mathrm{D}}},
$$

where $T$ is the total thrust over the rotor area, $A_{\mathrm{D}}=\pi R^{2}$ is the area of the rotor disk, and $R$ is the rotor radius. More details regarding both actuator-disk models are given in $\mathrm{Wu}$ and Porté-Agel (2011).

In the above-mentioned wind-turbine models, the blade-induced forces are distributed smoothly to avoid singular behaviour and numerical instability. In practice, these forces are distributed in a three-dimensional Gaussian manner by taking the convolution of the computed local load, $\mathbf{f}$, and a regularization kernel $\eta_{\epsilon}$ as shown below

$$
\begin{aligned}
\mathbf{f}^{\epsilon} & =\mathbf{f}^{\text {disk }} \otimes \eta_{\epsilon}, \\
\eta_{\epsilon} & =\frac{1}{\epsilon^{3} \pi^{3 / 2}} \exp \left[-\frac{\left(r_{\mathrm{p}}\right)^{2}}{\epsilon^{2}}\right],
\end{aligned}
$$

where $r_{\mathrm{p}}$ is the distance between grid points and points representing the actuator disk, and $\epsilon$ is a parameter that adjusts the distribution of the regularized load.

The effects of the nacelle and the turbine tower on the turbulent flow are modelled as drag forces $\mathbf{f}^{\text {nacelle }}$ and $\mathbf{f}^{\text {tower }}$, respectively, by using a formulation similar to the ADM-NR and a drag coefficient based on the specific geometry. As a result, the total immersed force $f_{i}$ associated with wind-turbine effects in the filtered momentum equation (Eq. 2) is given by

$$
f_{i}=\left(\mathbf{f}^{\epsilon}+\mathbf{f}^{\text {nacelle }}+\mathbf{f}^{\text {tower }}\right) \cdot \mathbf{e}_{i},
$$

where $\mathbf{e}_{i}$ is the unit vector in the $i$-th direction.

\section{Numerical Set-up}

In this study, we use a modified version of the LES code developed by Albertson and Parlange (1999), Porté-Agel et al. (2000), Stoll and Porté-Agel (2006), and Wu and Porté-Agel (2011). The Lagrangian scale-dependent dynamic model for the SGS stress is implemented in simulations of a neutrally-stratified boundary-layer flow through a wind farm installed over a horizontally-homogeneous flat surface. The turbine-induced forces are parametrized 
using the two above-mentioned actuator-disk models (ADM-NR and ADM-R). The main features of the code can be summarized as follows: the code uses a vertically staggered grid system with a uniform spacing for each direction, a second-order accurate Adams-Bashforth scheme for time advancement, and a mixed pseudospectral finite-difference scheme for spatial derivatives. These spatial derivatives are computed using a high-order pseudospectral scheme in the horizontal directions and a second-order finite-difference approximation in the vertical direction. As a result, the lateral boundary conditions are periodic and the top boundary condition is set up as a flux-free condition. The bottom boundary condition requires the calculation of the instantaneous (filtered) surface shear stress as a function of the velocity field at the lowest vertical grid point, which is accomplished through the application of Monin-Obukhov similarity theory. More details on the numerical method of the LES code can be found in Stoll and Porté-Agel (2006), Wu and Porté-Agel (2011), and PortéAgel et al. (2011). A brief description of the case study for model validation is given below.

The validation case study corresponds to a wind-tunnel wind-farm experiment performed by Chamorro and Porté-Agel (2011) in the St. Anthony Falls Laboratory ABL wind tunnel under neutral stratification. In that experiment, a turbulent boundary-layer flow was developed in the $16 \mathrm{~m} \times 1.7 \mathrm{~m} \times 1.7 \mathrm{~m}$ test section of the tunnel. At the downwind part of the test section, the flow had a free-stream velocity of $U_{\mathrm{f}} \approx 3.0 \mathrm{~m} \mathrm{~s}^{-1}$ and a boundary-layer depth of $\delta \approx 0.68 \mathrm{~m}$; the friction velocity and surface roughness length are $u_{*}=0.12 \mathrm{~m} \mathrm{~s}^{-1}$ and $z_{\mathrm{o}}=0.03 \mathrm{~mm}$, respectively. The wind farm had an aligned configuration and consisted of 30 miniature, horizontal-axis, three-bladed wind turbines arranged in 10 rows and three columns that were spaced $S_{x}=5 d$ apart in the streamwise direction and $S_{y}=4 d$ apart in the spanwise direction, where $d=0.150 \mathrm{~m}$ is the rotor diameter (see Fig. 2a). Each turbine consists of a three-bladed GWS/EP-6030 $\times 3$ rotor attached to a small DC generator motor at a hub height $\left(H_{\text {hub }}\right)$ of $0.125 \mathrm{~m}$. In the experiment, the high-resolution velocity measurements were collected with hot-wire anemometry for selected downwind locations at $x_{T i} / d=1$, 2, 3 and 4, where $x_{T i}$ is the relative streamwise distance from the $i$-th row of wind turbines and the subscript $i=1,2,3, \cdots 7$ and 10. All the measurements were taken on a vertical plane at zero span $(y=0)$ (shown as the vertical solid lines in Fig. 2a) for elevations ranging from the near-ground level $(z=0.01 \mathrm{~m})$ to $z=0.4 \mathrm{~m}$. At every location, streamwise and vertical velocity components were sampled at $2 \mathrm{kHz}$ for a measurement period of $60 \mathrm{sec}$. The normalized angular velocity distribution measured in the aligned wind farm is shown in Fig. 2b.

In this study, the effect of the wind-farm configuration on the flow is also investigated using LES. A staggered wind farm, where the even turbine rows are shifted laterally by $2 d$ with respect to the aligned layout, is selected. The angular velocity of the turbines in the staggered farm was measured (see Fig. 2b) using the same inflow condition with a free-stream velocity $U_{\mathrm{f}} \approx 3.0 \mathrm{~m} \mathrm{~s}^{-1}$. In the wind-tunnel experiments, the angular velocity of the turbines decreased in response to a reduction of the wind velocity due to wake effects. Based on the measured angular velocity, the tip speed ratio (TSR) for each turbine is between 4 and 5, which falls within a typical TSR range (3-10) of large-scale wind turbines.

To simulate the entire wind-farm wake, the horizontal computational domain spans a distance $L_{x}=72 d=10.8 \mathrm{~m}$ in the streamwise direction and $L_{y}=12 d=1.8 \mathrm{~m}$ in the spanwise direction. In the experiment, the boundary-layer depth $\delta$ grew slightly along the streamwise direction due to the increased effective surface roughness induced by the wind farm. To allow for this effect in the simulations, the computational domain has a vertical height $L_{z}$, which is slightly higher than the depth of the incoming boundary-layer flow. A constant streamwise pressure gradient is used to drive the flow within the boundary layer. Both Coriolis and 
(a)
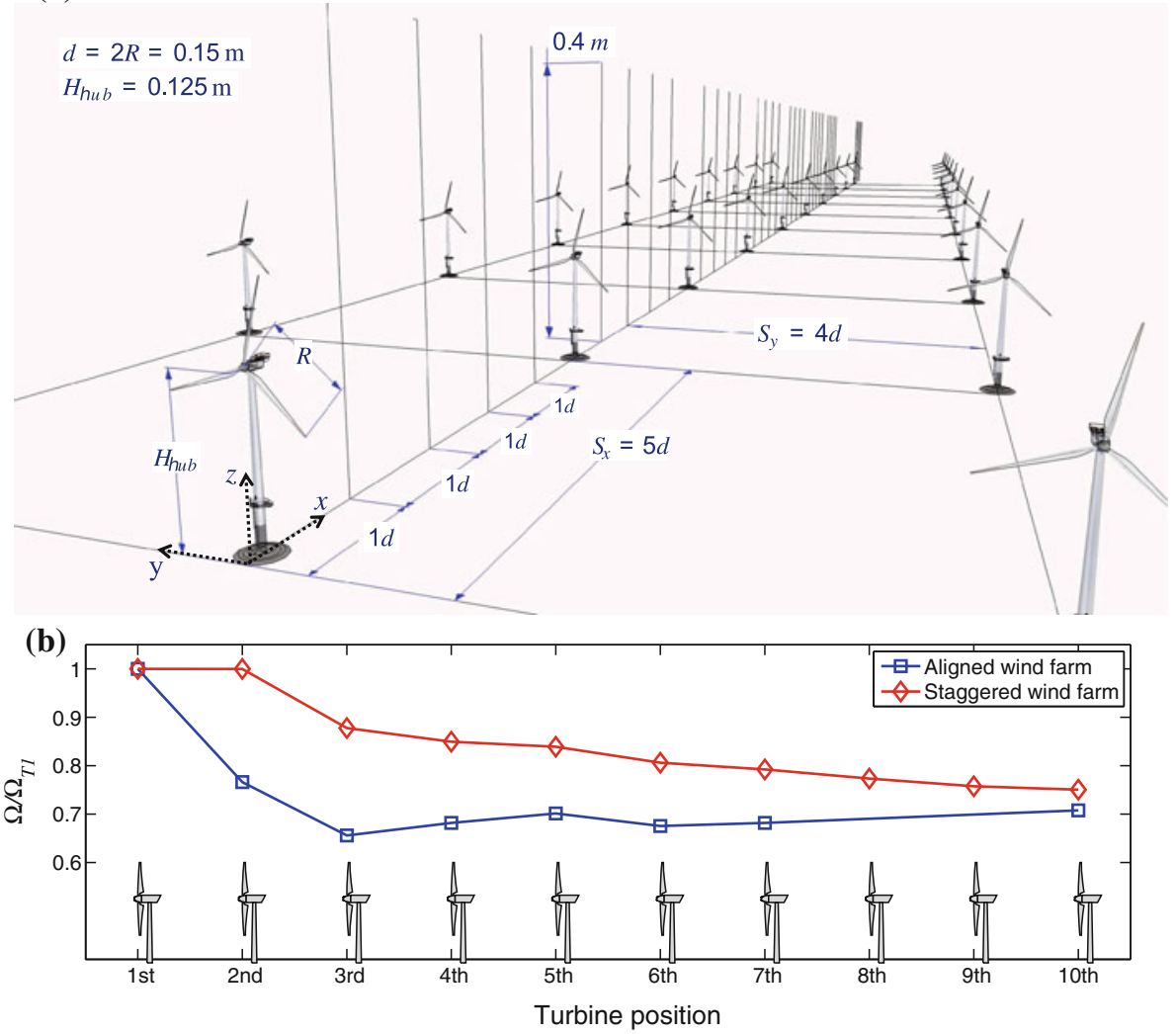

Fig. 2 a Schematic of the aligned wind-farm configuration. b Normalized measured angular velocity distribution of the wind turbines at different downwind positions

Table 1 Numerical set-up for neutrally-stratified ABL simulations with different grid resolutions

\begin{tabular}{lcrrrrrrrrr}
\hline Case & \multirow{2}{*}{$N_{x}$} & \multicolumn{1}{c}{$N_{y}$} & \multicolumn{1}{c}{$N_{z}$} & $\begin{array}{l}L_{x} \\
(\mathrm{~m})\end{array}$ & $\begin{array}{l}L_{y} \\
(\mathrm{~m})\end{array}$ & $\begin{array}{l}L_{z} \\
(\mathrm{~m})\end{array}$ & $\begin{array}{l}\delta \\
(\mathrm{m})\end{array}$ & $N_{t, y}$ & $N_{t, z}$ & $\Delta_{x}: \Delta_{y}: \Delta_{z}$ \\
\hline ADM-R1 & 180 & 60 & 30 & 10.8 & 1.8 & 0.870 & 0.68 & 5 & 5 & $2.0: 1.0: 1.0$ \\
ADM-R2 & 252 & 84 & 42 & 10.8 & 1.8 & 0.879 & 0.68 & 7 & 7 & $2.0: 1.0: 1.0$ \\
ADM-R3 & 324 & 108 & 54 & 10.8 & 1.8 & 0.875 & 0.68 & 9 & 9 & $2.0: 1.0: 1.0$ \\
ADM-R4 & 252 & 60 & 42 & 10.8 & 1.8 & 0.879 & 0.68 & 5 & 7 & $2.0: 1.4: 1.0$ \\
ADM-R5 & 648 & 108 & 108 & 10.8 & 1.8 & 0.892 & 0.68 & 9 & 18 & $2.0: 2.0: 1.0$
\end{tabular}

buoyant effects are neglected since the study focuses on a wind-tunnel boundary-layer flow with high Rossby number, $R_{\mathrm{O}} \approx 2,400$, under neutral stratification conditions. The domain is uniformly divided into $N_{x} \times N_{y} \times N_{z}$ grid points with a spatial resolution of $\Delta_{x} \times \Delta_{y} \times \Delta_{z}$ in the streamwise, spanwise and wall-normal directions, respectively. Five different spatial resolutions (see Table 1) are used to test the resolution sensitivity of the simulation results. In Table $1, N_{t, y}=d / \Delta_{y}$ and $N_{t, z}=d / \Delta_{z}$ denote the number of grid points covering the rotor diameter in the spanwise and vertical directions, respectively. 
The drag coefficients required to model the effects of the nacelle and the tower are based on the turbine geometry. In particular, the motor/generator has a cylindrical shape with a diameter $d_{\mathrm{m}}=0.015 \mathrm{~m}$ and a length $l_{\mathrm{m}}=0.03 \mathrm{~m}$. The turbine tower is also of a cylindrical shape with a diameter $d_{\mathrm{m}}=0.005 \mathrm{~m}$ and a length $l_{\mathrm{m}}=0.118 \mathrm{~m}$. The drag coefficient of bluff bodies of shape similar to typical cylinders varies between 0.8 and 1.2 (Schetz and Fuhs 1996; Kasmi and Masson 2008), depending on the their geometry and fineness ratio. For the case of the cylindrical nacelle and the turbine tower considered here, the drag coefficients of the nacelle $\left(C_{\mathrm{D} \text {,nac }}\right)$ and the turbine tower $\left(C_{\mathrm{D} \text {,tower }}\right)$ are set to 0.85 and 1.2 , respectively. In our simulations, the blade section is assumed to be a flat plate from root to tip. We adopt the data of lift and drag coefficients versus the angle of attack obtained from the experimental results of Sunada et al. (1997) for a flat plate at different Reynolds numbers. The radial variation of the chord length and the pitch angle has been presented in Wu and Porté-Agel (2011).

To avoid the turbine-induced wake flow affecting the flow upwind of the wind farm due to the periodic boundary conditions, a buffer zone upstream of the wind farm is employed to adjust the flow from the very-far-wake downwind condition to that of an undisturbed boundary-layer inflow condition. This inflow condition is obtained from a separate simulation of the boundary-layer flow corresponding to the upwind of the wind farm in the wind-tunnel experiment of Chamorro and Porté-Agel (2011). The use of a similar buffer region to impose the inflow boundary condition while maintaining the accuracy of pseudospectral LES codes has been successful in former studies of turbulent transport in urban street canopies (Tseng et al. 2006) as well as flow over a steep hill (Wan and Porté-Agel 2011) and flow through a stand-alone wind turbine (Wu and Porté-Agel 2011). All numerical simulations were run for a period (physical time) of more than $80 \mathrm{sec}$, and the flow statistics were computed during the last $60 \mathrm{sec}$, which corresponds to the measurement period and guarantees quasi-steady flow conditions as well as statistical convergence of the results presented below.

\section{Results}

\subsection{LES Validation}

In this section we present simulation results obtained from LES with the two actuator-disk models (ADM-R and ADM-NR) for the finest grid resolution $(648 \times 108 \times 108)$. The results are compared with the wind-tunnel measurements. In particular, we focus on the spatial distribution of three key turbulence statistics used to characterize the flow inside and above wind farms: the normalized time-averaged streamwise velocity $\bar{u} / \bar{u}_{\text {hub }}$, the streamwise turbulence intensity $\sigma_{u} / \bar{u}_{\text {hub }}$, and the kinematic shear stress $\tau_{x z}$ (resolved stress plus SGS stress). The overbar denotes a temporal average and $\bar{u}_{\text {hub }} \approx 2.6 \mathrm{~m} \mathrm{~s}^{-1}$ is the wind-farm inflow velocity at the hub height.

Figure 3 shows contours of the normalized time-averaged streamwise velocity obtained from the wind-tunnel experiment and the simulations with the ADM-R and the ADM-NR models on a $x-z$ vertical plane at the zero span $(y=0)$. To facilitate the quantitative comparison of the results, vertical profiles of the measured and simulated time-averaged streamwise velocity normalized by $\bar{u}_{\text {hub }}$ are shown in Fig. 4 for the selected downwind locations $x_{T i} / d=1$ to 4 (where the subscript $i=1$ to 7 and 10). As expected, the turbine wakes (regions of reduced mean velocity) are clearly visible behind each turbine. Also evident is the cumulative effect of multiple wakes, leading to the formation of a 'wind-farm wake' with two distinct regions: in the first region, below the top-tip level, the mean velocity deficit adjusts 

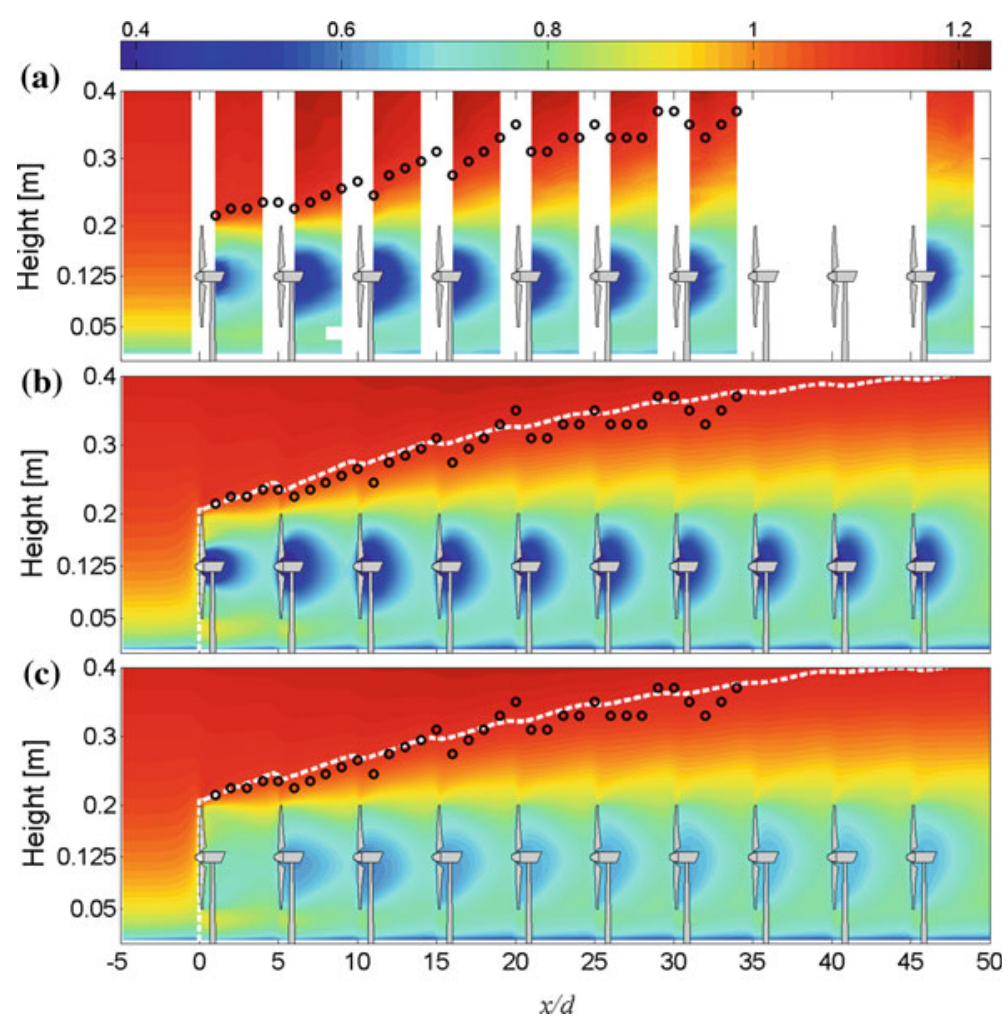

Fig. 3 Contours of the normalized time-averaged streamwise velocity $\bar{u} / \bar{u}_{\text {hub }}$ in the vertical plane at zero span $(y=0)$ ): wind-tunnel measurements (top), ADM-R (middle), ADM-NR (bottom). Circles in the three subplots denote the edge of the measured farm wake. White dashed lines in the subplots $\mathbf{b}$ and $\mathbf{c}$ denote the edge of the simulated farm wake obtained from the ADM-R and ADM-NR, respectively. The edge of the farm wake is defined as the height where the time-averaged streamwise velocity is $99 \%$ of the mean inflow velocity at that height

relatively rapidly and reaches an equilibrium after only two-three rows of wind turbines. This is consistent with field observations showing that the power output from operational offshore aligned wind farms decreases significantly (with respect to the first row) for the second and, to a lesser extent, the third row of turbines, while it remains relatively unchanged after that (e.g., Barthelmie et al. 2007). In the second region, above the turbines, the flow experiences a larger downwind variation as the cumulative farm wake expands. The edge of the farm wake, defined here as the height where the time-averaged wake velocity is $99 \%$ of the mean inflow velocity at that height, is shown in Fig. 3. The location of the simulated wake edge is very similar for both turbine models and it is in good agreement with the measurements. The wake edge grows with downwind distance and reaches a height of about $0.4 \mathrm{~m}$ (twice the turbine height) behind the tenth row of turbines $(x / d \geq 45)$.

The simulation results in Figs. 3 and 4 show clear differences between the predictions of the wind velocity deficit by the two wind-turbine models. In particular, the LES with the ADM-R produces velocity profiles that are in good agreement with the measurements throughout the wind farm. In contrast, the ADM-NR clearly overpredicts the mean velocity (i.e., it underpredicts the velocity deficit) in the wake behind each turbine. This is consistent 

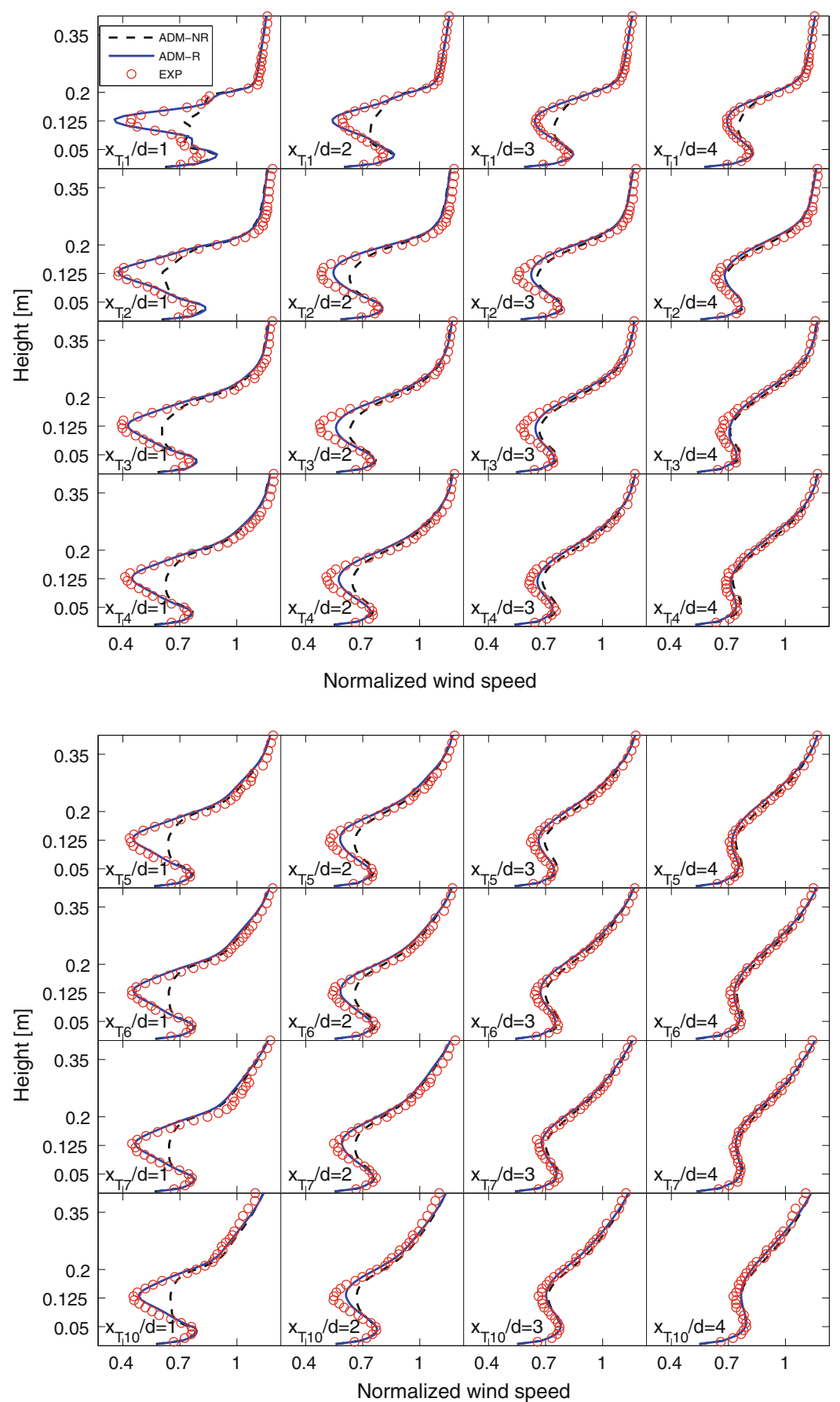

Fig. 4 Comparison of vertical profiles of the normalized time-averaged streamwise velocity $\bar{u} / \bar{u}_{\text {hub }}$ : windtunnel measurements (open circle), ADM-R model (solid line) and ADM-NR model (dashed line) 

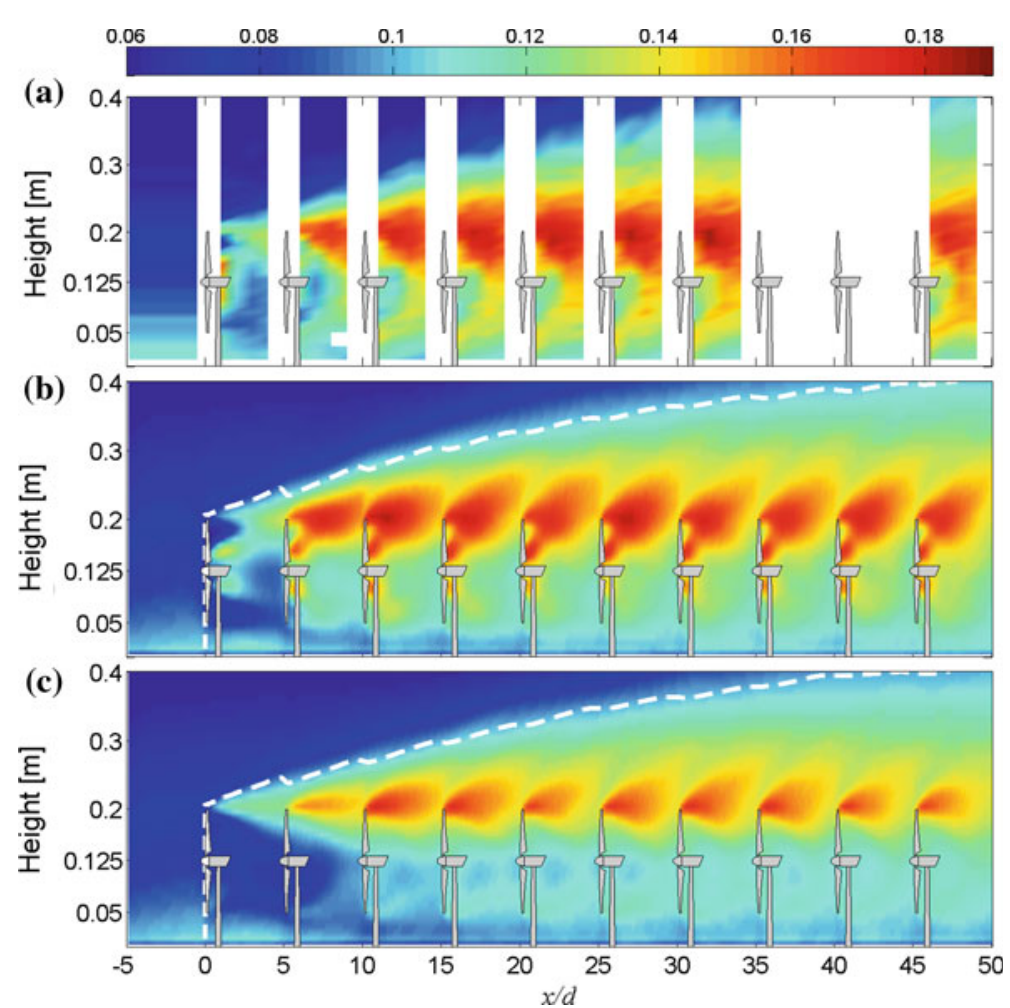

Fig. 5 Contours of the streamwise turbulence intensity $\sigma_{u} / \bar{u}_{\text {hub }}$ in the vertical plane at zero span $(y=0)$ : wind-tunnel measurements (top), ADM-R (middle), ADM-NR (bottom). White dashed lines in the subplots b and $\mathbf{c}$ denote the edge of the simulated farm wake obtained from the ADM-R and ADM-NR models, respectively

with the previous simulations of the wake of a stand-alone turbine presented by Wu and PortéAgel (2011) and Porté-Agel et al. (2011). It should be noted that, as discussed in Sect. 2, the thrust coefficient $C_{\mathrm{T}}$ used for each turbine in the ADM-NR is obtained based on the overall thrust force computed using the BEM theory in the ADM-R. Consequently, the failure of the ADM-NR model to reproduce the velocity magnitude in the wake regions is attributed to the limitations of two major assumptions made in the ADM-NR (but not in the ADM-R): (a) the turbine-induced rotation effect is ignored, and (b) the axial thrust force is uniformly distributed over the rotor disk area, thus ignoring the radial variation of the force. As pointed out by Wu and Porté-Agel (2011), the latter assumption has the stronger effect of the two.

Despite the obvious differences between the simulated near-wake mean velocities, in Fig. 4 we observe that the two turbine models predict almost identical (and very close to the measurements) velocity profiles at a distance of four rotor diameters downstream of the turbines after the second row of the farm turbines (i.e., $x_{T i} / d=4$, where $i$ is from 2 to 7 and 10). However, the difference between the model results is still evident at the same distance behind the first row of turbines $\left(x_{T 1} / d=4\right)$, which is consistent with simulation results of the wake of a stand-alone wind turbine (Wu and Porté-Agel 2011). This result seems to indicate that, inside the wind farm, the relatively faster turbine-wake recovery, associated 

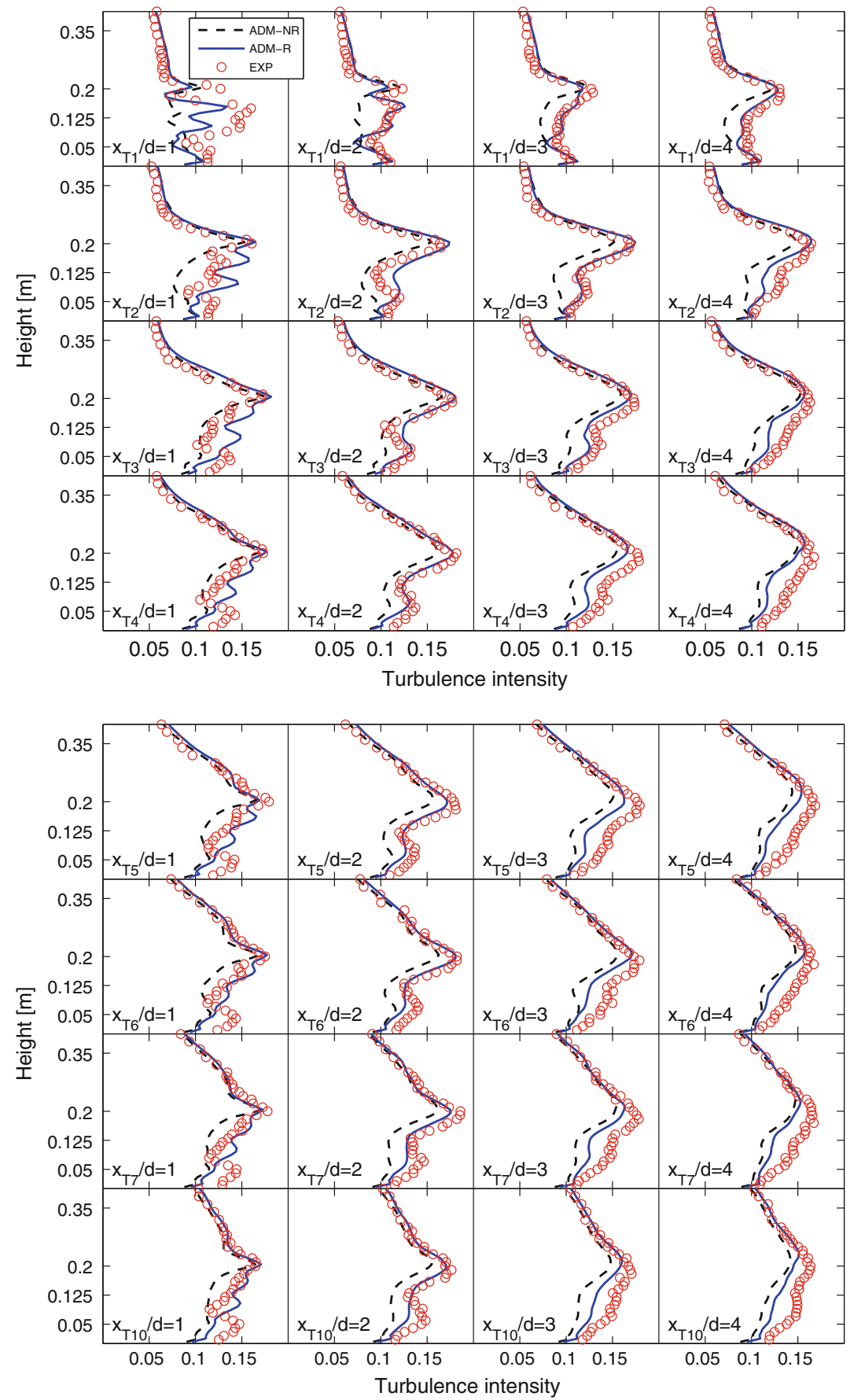

Fig. 6 Comparison of vertical profiles of the streamwise turbulence intensity $\sigma_{u} / \bar{u}_{\text {hub }}$ : wind-tunnel measurements (open circle), ADM-R model (solid line) and ADM-NR model (dashed line) 
with higher background turbulence levels (discussed below), helps reduce the differences produced by the different thrust force distributions in the two models.

Figure 5 displays contours of the streamwise turbulence intensity obtained from the windtunnel experiment and the simulations on the same $x-z$ vertical plane at the zero span $(y=0)$. Again, vertical profiles of the measured and simulated streamwise turbulence intensity are shown in Fig. 6 for the same selected downwind locations $x_{T i} / d=1$ to 4 (where the subscript $i=1$ to 7 and 10). Due to the cumulative effect of the multiple wakes from upstream wind turbines, the maximum level of the turbulence intensity found behind the wind turbines increases substantially in the wakes behind the first four rows of turbines, and reaches a plateau after the fifth row. That maximum turbulence intensity is found behind each turbine at the top-tip level. This is due to the high mean shear (see Fig. 4) and associated mechanical (shear) turbulence production in that region. In particular, a peak of turbulence is found at approximately three rotor diameters behind each of the turbines, except for the first one. This is due to the fact that the inflow to the first row of wind turbines is much less turbulent, leading to a slower recovery of the wake (due to less efficient mixing with the surrounding flow), compared with the wakes of the other turbines. This also explains why the turbulence intensity peak is found further downwind from the turbine in the case of a stand-alone wind turbine (e.g., Wu and Porté-Agel 2011; Porté-Agel et al. 2011).

The distribution of the simulated turbulence intensity obtained with the two turbine models (ADM-NR and ADM-R) shows a similar qualitative behaviour as the one reported in the experiment. However, significant differences are found in the ability of the two models to match quantitatively the measured turbulence intensity levels. The magnitude of the turbulence intensity obtained with the ADM-R, and particularly its maximum value at the top-tip level, is found to be in acceptable agreement with the wind-tunnel measurements. The ADM$\mathrm{NR}$, however, tends to systematically underestimate the peak of turbulence intensity behind most of the turbines. Below the top-tip height, and further than two rotor diameters downwind of the turbines, both models underestimate the turbulence intensity, with a slightly worse prediction from the ADM-NR model.

Figure 7 displays contours of the kinematic shear stress $\tau_{x z}$ obtained from the wind-tunnel experiment and simulations on a $x-z$ vertical plane at the zero span $(y=0)$. From the measurements, it is clear that the turbines introduce a positive and a negative shear stress in the wakes above and below the turbine hub height, respectively. The cumulative effect of the multiple wakes from upstream turbines results in an increase in the magnitude of the kinematic shear stress in the first three rows of wind turbines, reaching a plateau after the fourth row. In the simulations, both wind-turbine models are able to account for that qualitative behaviour, though important quantitative differences are observed. In general, the ADM-R model yields a better prediction of the maximum kinematic shear stresses in the wakes above the hub height behind each turbine. However, both wind-turbine models are unable to predict accurately the turbulent stress below the hub height, with the ADM-R underestimating and the ADM-NR overestimating the magnitude of the shear stress, respectively.

Of special interest for the optimization of computational resources is to determine the coarsest resolution at which the proposed LES framework yields reasonable results. In order to study the resolution sensitivity of the computational results, a series of simulations are performed with different grid resolutions using the ADM-R. The grid resolutions considered here have three different aspect ratios as shown in Table 1. Vertical profiles of the normalized streamwise time-averaged velocity and the streamwise turbulence intensity (resolved part) are presented in Fig. 8 for the simulations using the four coarser grid resolutions (ADM-R1 to ADR-R4 in Table 1). The simulation results using the finest grid resolution (ADM-R5) have already been plotted in Figs. 4 and 6. In Fig. 8, the vertical profiles of streamwise velocity 

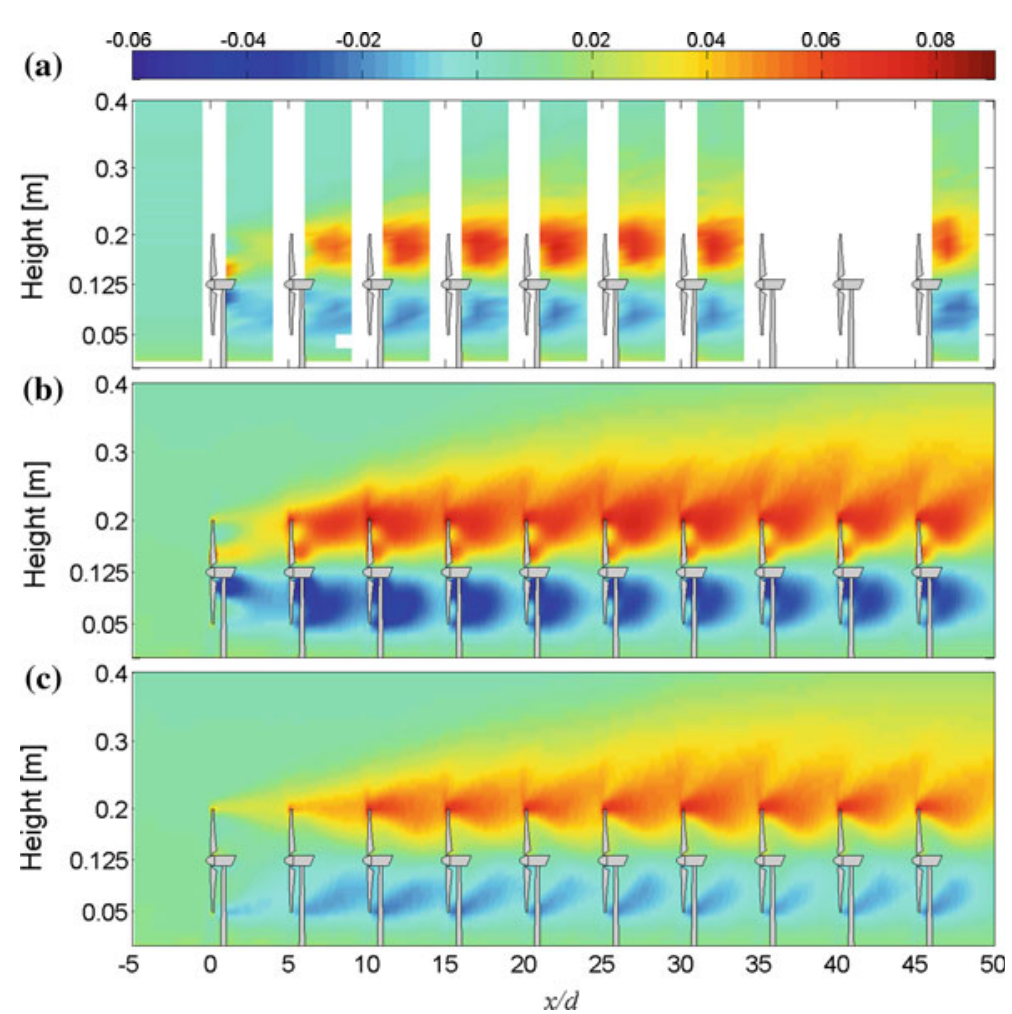

Fig. 7 Contours of the kinematic shear stress $\tau_{x z}\left(\mathrm{~m}^{2} \mathrm{~s}^{-2}\right)$ in the vertical plane at zero span $(y=0)$ : wind-tunnel measurements (top), ADM-R model (middle), ADM-NR model (bottom)

show a very small resolution dependence for the range of resolutions tested. However, the results obtained from the ADM-R1 (with 5 by 5 grid points covering the rotor diameter in the both spanwise and vertical directions) start to show an underestimation of the wake turbulence intensity, particularly at the top-tip level, with respect to the higher-resolution simulations (ADM-R2 and ADM-R3) and the experimental results. The outcome of these resolution sensitivity tests suggests that, for the range of grid aspect ratios considered here, the LES framework yields results that are quite accurate and have little resolution dependence as long as at least seven points are used to cover the rotor diameter in the vertical direction, and five points in the spanwise direction. It should be noted that these grid resolution sensitivity results have been obtained under neutral stratification, and they could change slightly under different stability conditions.

The dynamically-calculated value of the model coefficient $C_{\mathrm{S}}$ obtained using the Lagrangian scale-dependent dynamic model is presented in Fig. 9. As expected, the model coefficient decreases near the surface and also near the edge of the farm wake (starting at the top-tip height of the first turbine), in order to account for the reduction in the characteristic scale of the turbulence associated with the increased shear (results not shown here) in those regions. Wu and Porté-Agel (2011) presented a similar trend of the model coefficient in the wake of a stand-alone wind turbine. Overall, these results, together with the reasonable flow prediction, show that the Lagrangian scale-dependent dynamic model can account for the effect of 

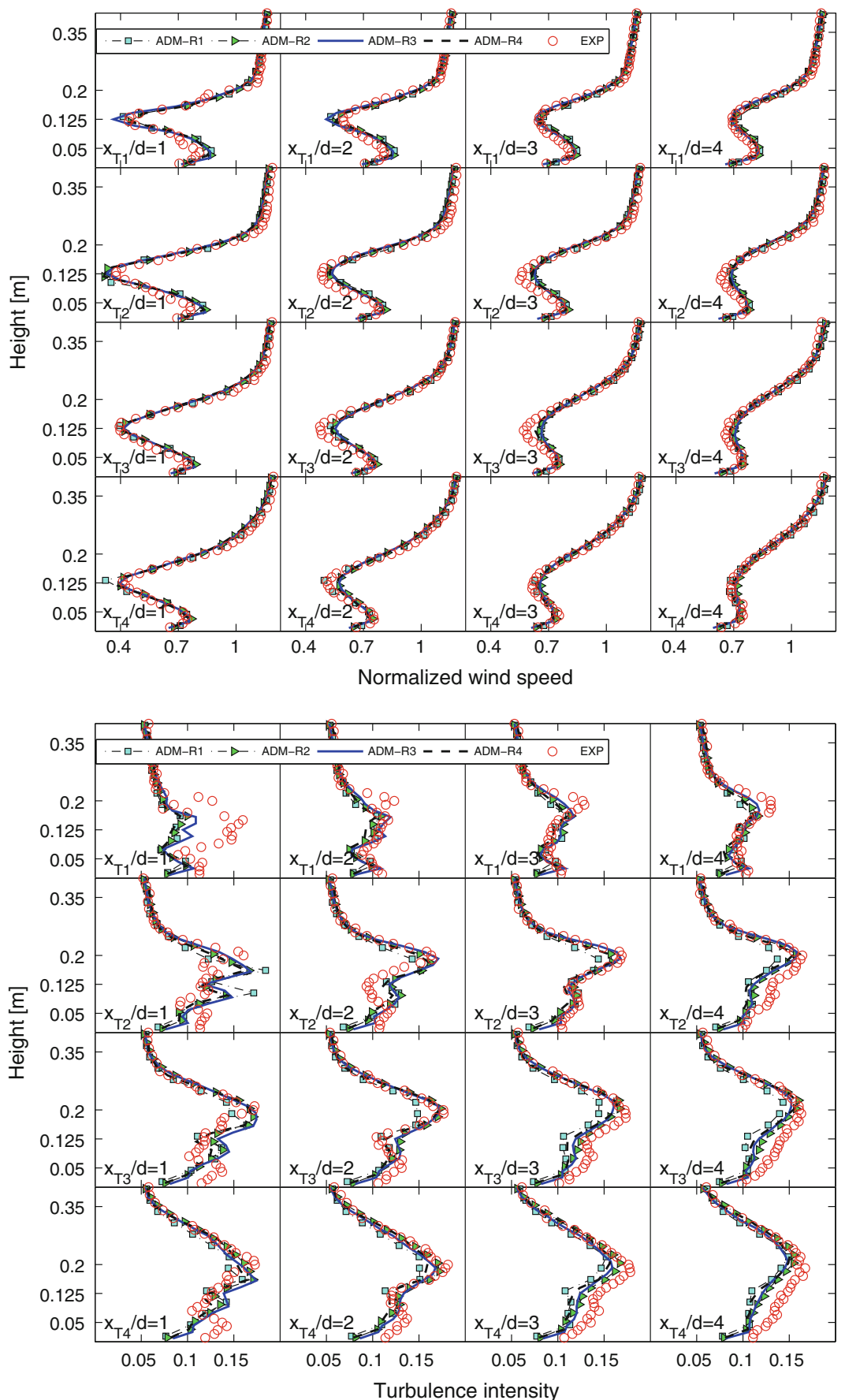

Fig. 8 Comparison of vertical profiles of the normalized time-averaged streamwise velocity $\bar{u} / \bar{u}_{\text {hub }}($ top $)$ and the streamwise turbulence intensity $\sigma_{u} / \bar{u}_{h u b}$ (bottom): wind-tunnel measurements (open circle), ADM-R1 (square), ADM-R2 (triangle), ADM-R3 (solid line) and ADM-R4 (dashed line) 


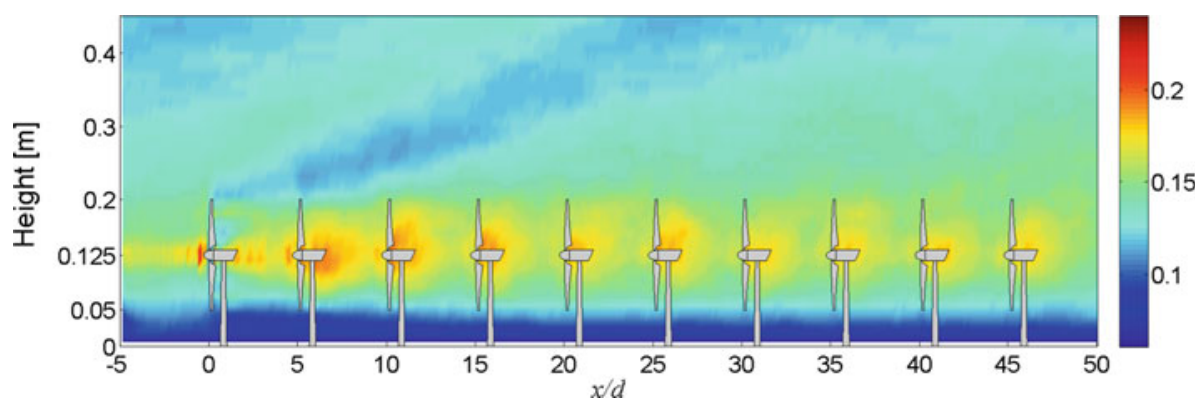

Fig. 9 Contour of the Smagorinsky coefficient $C_{\mathrm{S}}$ obtained with the Lagrangian scale-dependent dynamic model as well as the ADM-R model

increased shear and local anisotropy of the flow on the reduction of the characteristic scales of the turbulence by dynamically (without any tuning) calculating the model coefficient.

\subsection{Wind-Farm Layout Effects}

After validation of the LES framework for simulation of turbulent boundary-layer flow through an aligned wind farm, here we use the LES to study the effect of wind-farm layout on the flow. To achieve that, a new simulation is set up that uses the same incoming neutral boundary-layer flow as the validation case, with the only difference that now the 30 wind turbines are arranged in a staggered layout such that the even turbine rows are shifted laterally by $2 d$ with respect to the aligned configuration. This guarantees the same overall farm area and, consequently, the same density of turbines. The numerical set-up in the highestresolution (ADM-R5) case is adopted in the simulation, except for the horizontal position and the angular velocity of the turbines. The turbine angular velocity distribution measured in the staggered wind farm (see Fig. 2b) is specified in the simulation for the BEM calculation used in the ADM-R model. A comparison of the LES results for the aligned and staggered wind farms is presented next.

Figure 10 shows contour plots of the normalized time-averaged streamwise velocity $\bar{u} / \bar{u}_{\text {hub }}$ (Fig. 10a), the streamwise turbulence intensity $\sigma_{u} / \bar{u}_{\text {hub }}$ (Fig. 10b), and the kinematic shear stress $\tau_{x z}$ (Fig. 10c) on a horizontal $x-y$ plane at the turbine hub height in the aligned and staggered wind farms. The same flow statistics are plotted in Fig. 11 on vertical $y-z$ planes located $3 d$ downwind of the third and the ninth rows of wind turbines in both the aligned and the staggered cases. From both figures, it is clear that the farm layout has a strong effect on the structure of the cumulative wakes and, consequently, on the distribution of the different turbulence statistics. In the aligned case, the turbine wake regions are centred around the turbine rows and grow radially with distance downwind, only interacting laterally after approximately the eighth row. In the case of the staggered wind farm, lateral wake interactions are obvious even after the third row of turbines due to the fact that the wind farm offers a larger 'frontal area' to the incoming flow. Moreover, since the effective distance between downwind turbines is now $10 d$, the wakes have a longer distance to recover before the next turbine, which results in a higher efficiency of the turbines (i.e., faster rotating speed) in extracting momentum from the flow, compared with the aligned counterpart. This explains the higher angular velocity of the turbines and the more uniform distribution of the velocity within the wind farm (Figs. 10a, 11a). According to the 1-D momentum theory, we can write the rotor power $(P)$ as proportional to the cube of the turbine angular velocity (i.e., $P \propto \Omega^{3}$ ). 


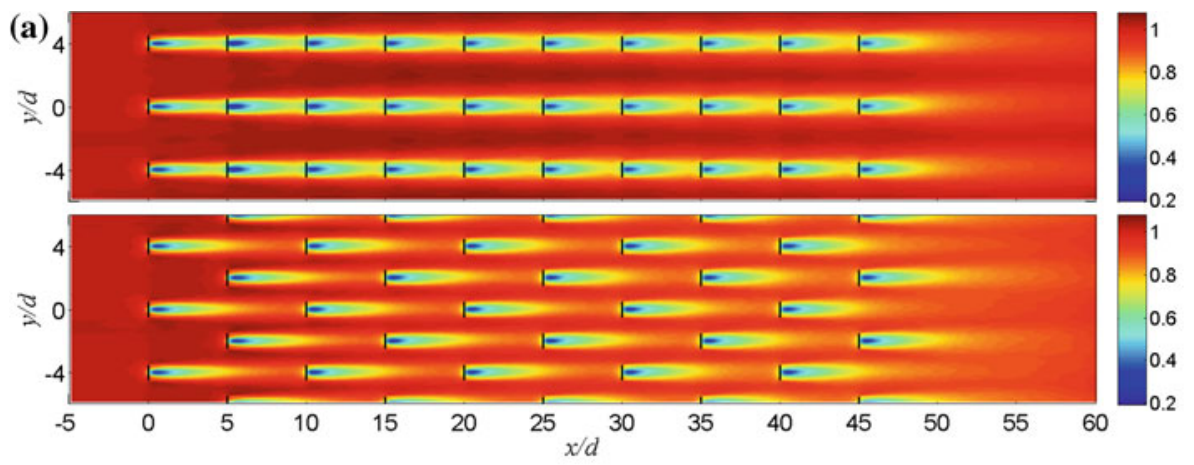

(b)
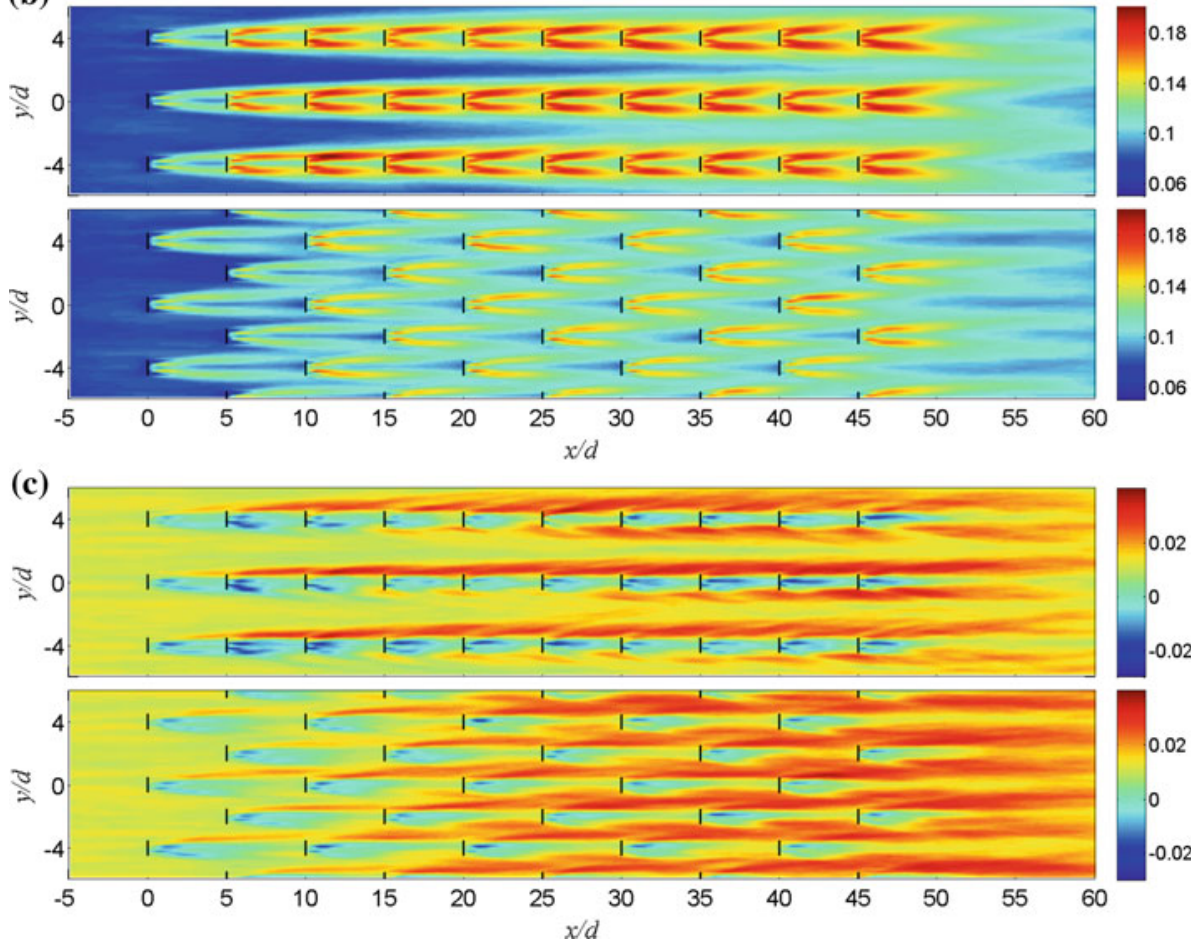

Fig. 10 Contours of a normalized time-averaged streamwise velocity $\bar{u} / \bar{u}_{\text {hub }}$, b streamwise turbulence intensity $\sigma_{u} / \bar{u}_{\text {hub }}$, and $\mathbf{c}$ kinematic shear stress $\tau_{x z}\left(\mathrm{~m}^{2} \mathrm{~s}^{-2}\right)$ on the horizontal $x-y$ plane at the turbine hub height in the aligned and staggered wind farms. Vertical black lines denote the position of the wind-turbine disks

Based on this simple relation, we find that the total power extracted by the rotors in the staggered wind farm considered here is about $64 \%$ larger than that in the aligned farm.

Important differences between the two layouts are also found in the turbulence intensity distribution. The enhancement of turbulence intensity and, consequently, the potential negative impacts of the associated fatigue loads, are much higher in the aligned wind farm. In the case of the staggered wind farm, the distance between 'immediately downwind' turbines is longer, which allows for the turbulence to dissipate to lower levels before reaching the next downwind turbine, thus reducing the cumulative turbulence enhancement effect. It should 

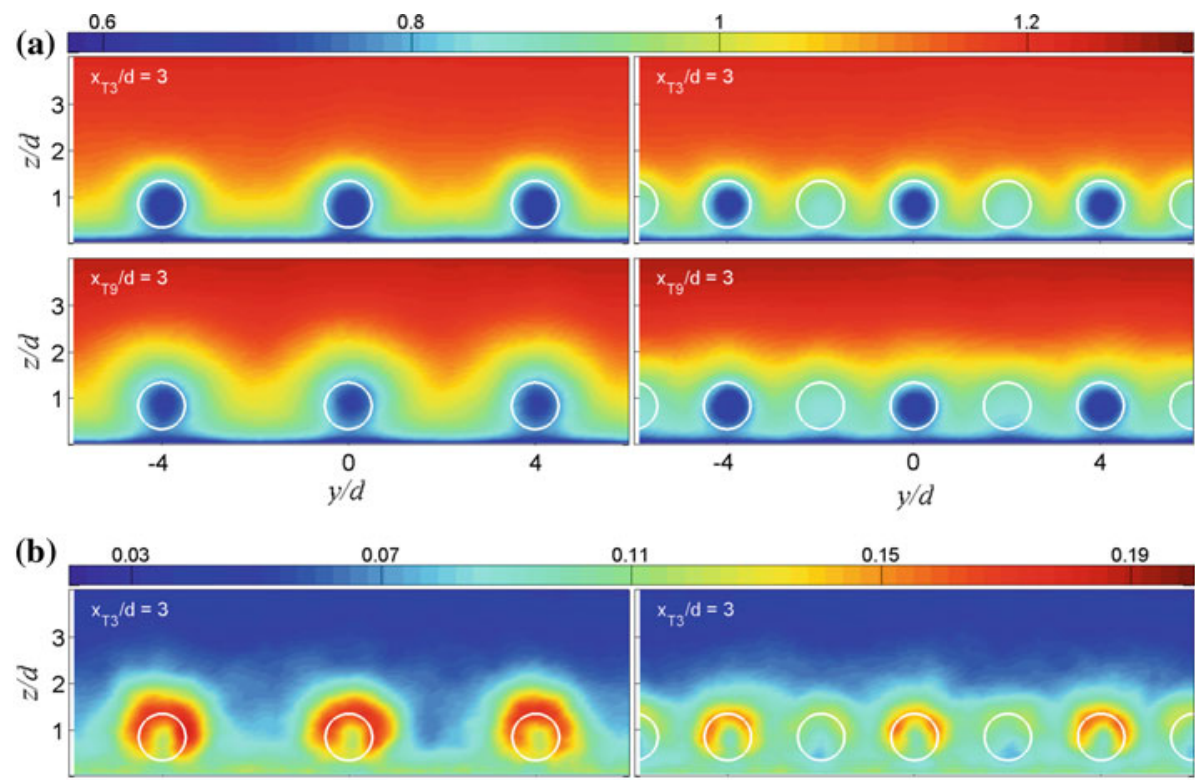

0.15

0.19

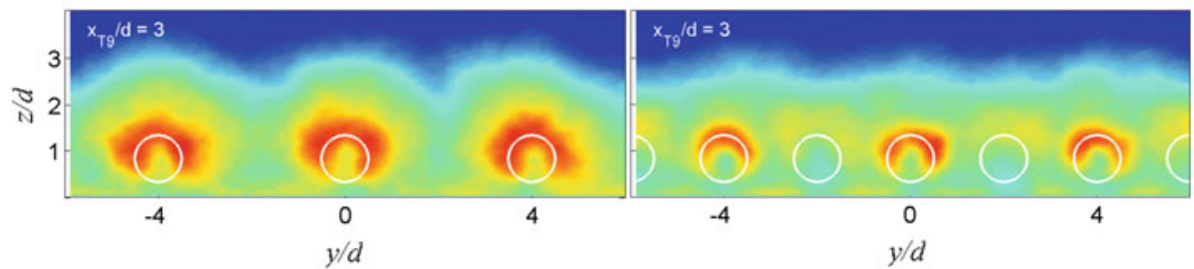

(c)
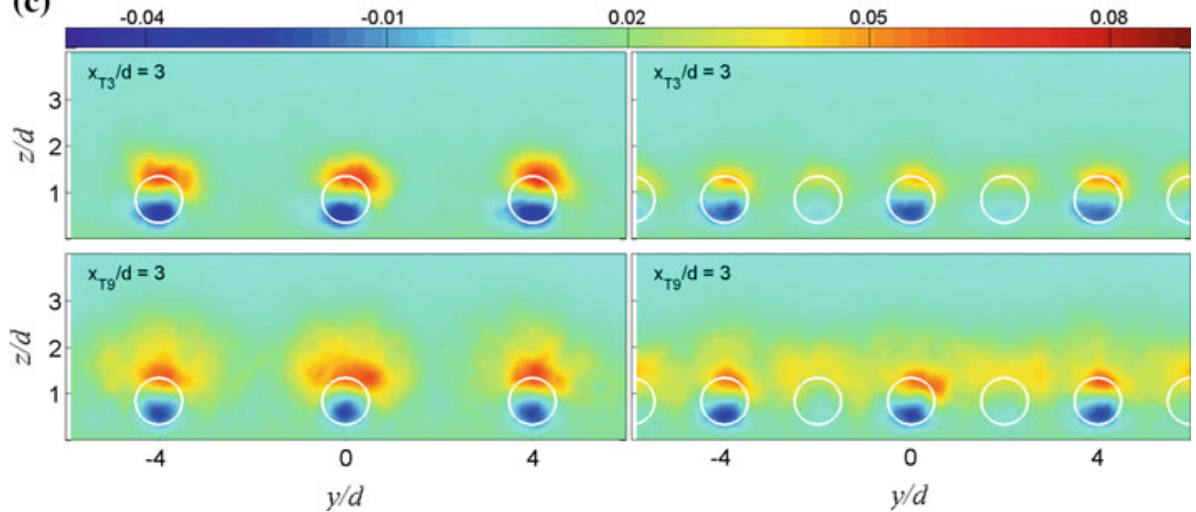

Fig. 11 Contours of a normalized time-averaged streamwise velocity $\bar{u} / \bar{u}_{\text {hub }}, \mathbf{b}$ streamwise turbulence intensity $\sigma_{u} / \bar{u}_{\text {hub }}$, and $\mathbf{c}$ kinematic shear stress $\tau_{x z}\left(\mathrm{~m}^{2} \mathrm{~s}^{-2}\right)$ in a vertical $y-z$ plane at $3 d$ downstream behind the third and ninth turbines in the aligned (left panel) and staggered (right panel) wind farms. The rotor regions are indicated with white circles with solid line

be noted that, as shown in Fig. 11b, the maximum turbulence intensity region corresponds to a U-shaped area at the rotor edge and above hub height, where the local shear and associated production of kinetic energy are high. This is consistent with findings from previous laboratory and numerical studies (Chamorro and Porté-Agel 2009, 2011; Porté-Agel et al. 

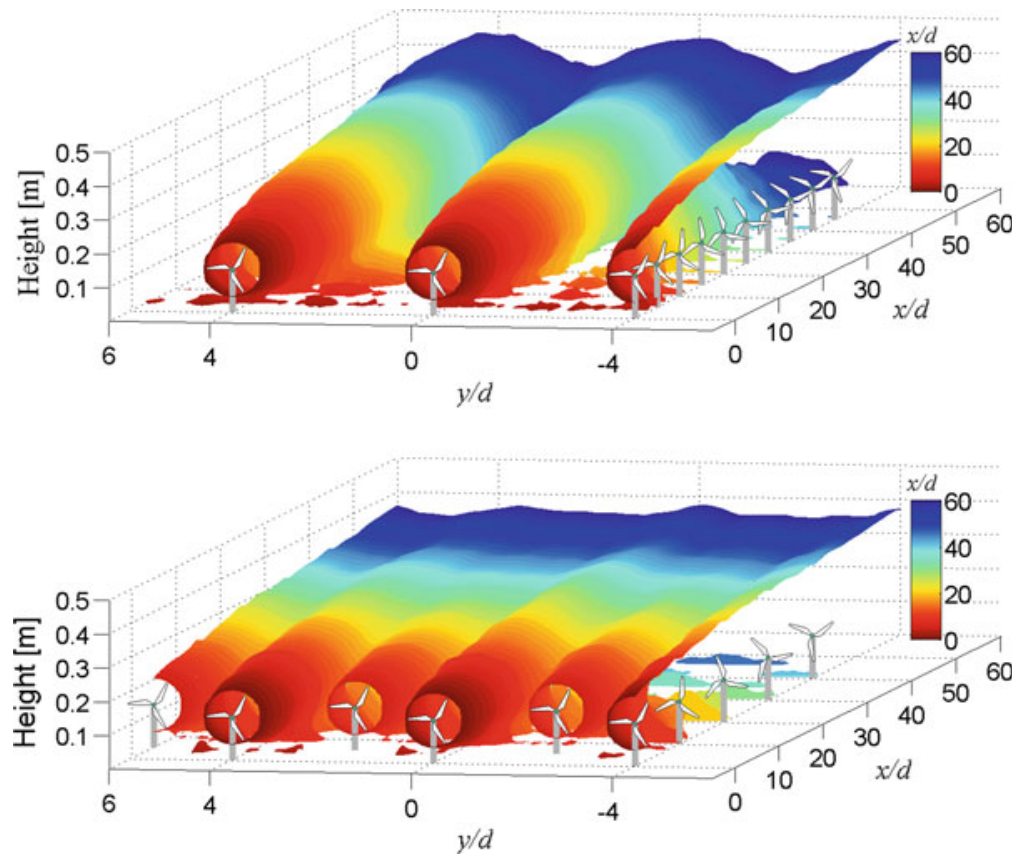

Fig. 12 Isosurface of internal wake layer distribution: aligned (top) and staggered (bottom) wind-farm configurations

2011; Wu and Porté-Agel 2011; Zhang et al. 2012). It is important to note that the maximum enhancement of turbulence intensity is not found at hub height, as is often assumed in simple models used to estimate wake turbulence inside wind farms (e.g., International Standard IEC 61400-1 2005).

In order to further illustrate the growth of the cumulated farm wakes and their dependence on farm layout, Fig. 12 depicts a three-dimensional representation of the distribution of the simulated wake edge over the aligned and staggered wind farms. The wake edge is defined as the location where the time-averaged wake velocity is $99 \%$ of the mean flow velocity at height. From this figure, it is obvious that in the aligned wind farm there is no lateral interaction between the turbines until the eighth row of turbines. In contrast, the wakes merge relatively soon, leading to a more uniform spanwise distribution of the wake edge. As a result, the growth of the cumulated farm wake resembles more a classical 'internal boundary layer' in the case of the staggered wind farm than in the case of the aligned wind farm.

Next, we study the effect of the wind-farm layout on the area-averaged streamwise velocity and the associated wind-farm effective roughness. The latter is an important parameter used to represent wind farms in large-scale atmospheric models. In order to understand the longitudinal variation of the mean velocity, we use averages in time and over a horizontal plane that spans the entire spanwise size of the computational domain (i.e., 12 rotor diameters) and five rotor diameters behind each row of wind turbines in the streamwise direction. This horizontal spatial average is denoted as \langle\rangle$_{T i}^{H}$ for the $i$-th row of turbines. Vertical profiles of the horizontally-averaged streamwise velocity for both aligned and staggered wind farms are shown in Fig. 13 for four selected turbine locations (T2, T4, T6 and T9), together with the inflow profiles. From Fig. 13a, b, it is clear that the mean velocity adjustment is 

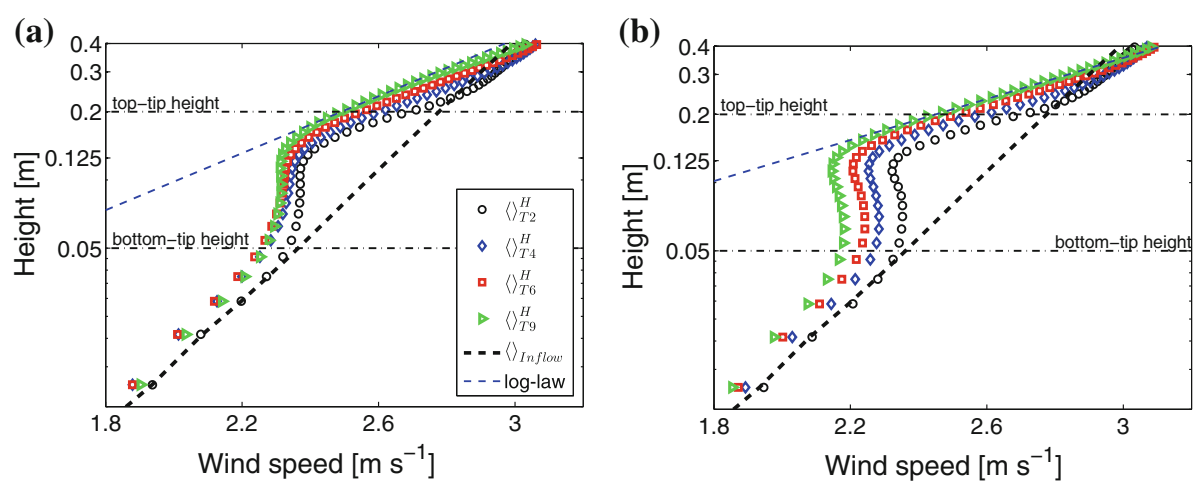

Fig. 13 Vertical profiles of horizontally-averaged streamwise velocity in the $\mathbf{a}$ aligned and $\mathbf{b}$ staggered wind farms. \langle\rangle$_{T i}^{\mathrm{H}}$ denotes an average over a horizontal plane (behind the $i$-th row of turbines) within $x_{T i} / d=0$ to 5 and $y / d=-6$ to 6 . \langle\rangle$_{\text {Inflow }}$ denotes a horizontal spatial average at inflow position

also affected by the farm layout. For example, below the hub height, the average velocity shows little change after the fourth row of turbines in the aligned farm. This is in contrast to the staggered farm flow, for which the mean velocity continues changing (decreasing) with downwind distance even at the tenth row of turbines. This behaviour is consistent with the reduction of angular velocity at the turbines shown in Fig. 2b. Above the wind farm, the mean velocity profiles are found to be logarithmic. For both configurations, the mean velocity does not reach an equilibrium due to the fact that the turbine wakes are still growing radially with downwind distance. It is important to note also that the maximum area-averaged turbulent shear stress, which is found at the top-tip level, does not completely adjust after ten rows.

As mentioned above, wind farms are often represented as increased aerodynamic roughness in large-scale atmospheric models. In previous studies, three analytical models have been proposed by Lettau (1969), Frandsen (1992) and Calaf et al. (2010) to quantify the effective wind-farm aerodynamic roughness. The three models can be summarized as follows:

- Lettau (1969)

$$
z_{\mathrm{o}, \mathrm{L}}=\frac{H_{\mathrm{T}} \pi}{8 s_{x} s_{y}}
$$

- Frandsen (1992)

$$
z_{0, \mathrm{~F}}=H_{\mathrm{T}} \exp \left[-\frac{\kappa}{\sqrt{\frac{1}{2} c_{\mathrm{ft}}+\left[\frac{\kappa}{\ln \left(\left(H_{\mathrm{T}}\right) / z_{\mathrm{o}}\right)}\right]^{2}}}\right],
$$

- Calaf et al. (2010)

$$
\begin{aligned}
z_{\mathrm{o}, \mathrm{C}} & =H_{\mathrm{T}}\left(1+\frac{d}{2 H_{\mathrm{T}}}\right)^{\frac{v_{\mathrm{w}}^{*}}{\left(1+v_{\mathrm{w}}^{*}\right)}} \\
& \times \exp \left(-\left[\frac{c_{\mathrm{ft}}}{2 \kappa^{2}}+\left(\ln \left[\frac{H_{\mathrm{T}}}{z_{\mathrm{o}}}\left(1-\frac{d}{2 H_{\mathrm{T}}}\right)^{\frac{v_{\mathrm{w}}^{*}}{\left(1+v_{\mathrm{w}}^{*}\right)}}\right]\right)^{-2}\right]^{-\frac{1}{2}}\right),
\end{aligned}
$$


(a)

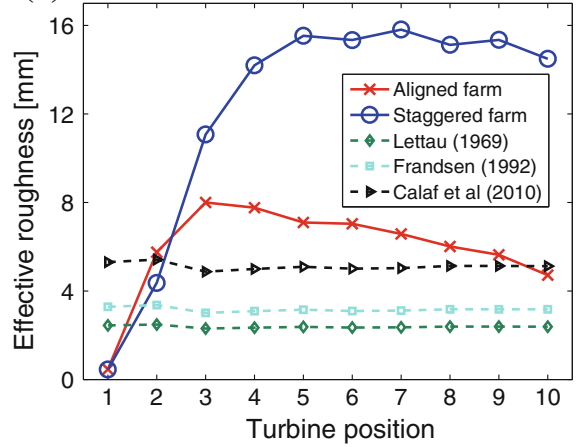

(b)

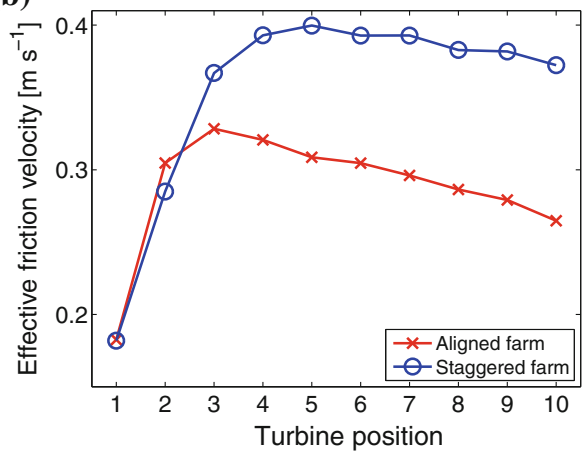

Fig. 14 Distribution of effective wind-farm a roughness parameter $z_{0}^{\text {eff }}$ and $\mathbf{b}$ friction velocity $u_{*}^{\text {eff }}$
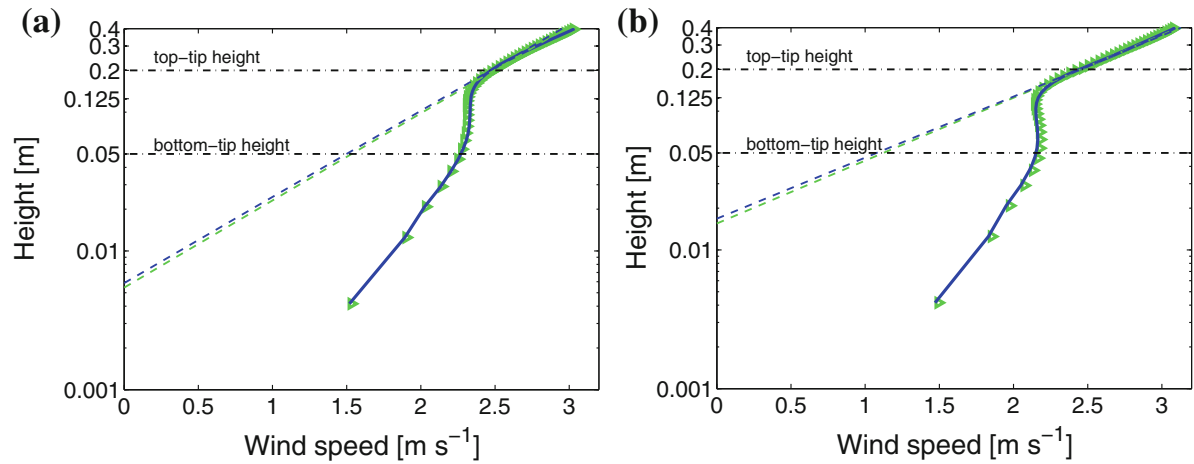

Fig. 15 Vertical profiles of horizontally-averaged streamwise velocity in the $\mathbf{a}$ aligned and $\mathbf{b}$ staggered wind farms. Green triangles denote an averaged velocity profile $\langle\bar{u}\rangle_{T 9}^{\mathrm{H}}$, and the blue solid line represents a velocity profile $\langle\bar{u}\rangle_{T 9}^{\text {Span }}$ averaged over four spanwise locations $(y / d=-1,0,1$ and 2$)$ at a streamwise location of $x_{T 9} / d=3$. Green and blue dashed lines are the results from a log-law regression analysis of the averaged velocity profiles $\langle\bar{u}\rangle_{T 9}^{\mathrm{H}}$ and $\langle\bar{u}\rangle_{T 9}^{\mathrm{Span}}$ at a vertical elevation ranging from $z=0.2 \mathrm{~m}$ to $z=0.25 \mathrm{~m}$, respectively

where $c_{\mathrm{ft}}=\left(\pi C_{\mathrm{T}}\right) /\left(4 s_{x} s_{y}\right), v_{\mathrm{w}}^{*}=\left(\sqrt{c_{\mathrm{ft}} / 2}\langle\bar{u}\rangle d\right) /\left(\kappa u_{*} H_{\mathrm{hub}}\right), s_{x}$ and $s_{y}$ are the spacings between wind turbines in the streamwise and spanwise directions, respectively, $H_{\mathrm{T}}=$ $H_{\text {hub }}+R$ is the turbine height, $\langle\bar{u}\rangle$ is the mean velocity at the turbine height.

In this study, as shown in Fig. 14, these three models are tested by comparing their prediction with the aerodynamic roughness obtained by fitting a logarithmic law to the vertical profiles of the mean simulated streamwise velocity profiles in the range of heights between $z=0.2 \mathrm{~m}=1.6 H_{\text {hub }}$ and $z=0.25 \mathrm{~m}=2 H_{\text {hub }}$, where a good log-law fit is obtained (regression coefficient larger than 0.99 ) for both wind-farm configurations. In this figure, the adjustment of both the effective wind-farm roughness and friction velocity with respect to the turbines is associated with the formation of the multiple wakes in the two wind farms, which indicates that the wakes in the aligned farm are still developing (compared with the staggered case).

The results of the LES simulations can also provide valuable information for experimentalists in order to optimize measurement strategies in wind farms, both in the wind tunnel and in the field. For example, in a recent wind-tunnel experiment using similar miniature wind turbines in aligned and staggered wind-farm layouts, Markfort et al. (2012) used four vertical 
profiles at four spanwise locations $(y / d=-1,0,1$ and 2) and at a streamwise location of $x_{T 9} / d=3$ to characterize the mean velocity profile of the wind farm behind the ninth row of wind turbines. Figure 15 shows the simulated mean velocity profile (from our simulations) obtained using those locations, and compares it with the overall average $\langle\bar{u}\rangle_{T 9}^{H}$ obtained using the full velocity field. From this, it can be concluded that the mean velocity profile obtained using carefully selected locations in the wake region can provide reliable estimates of the overall mean velocity inside and above the wind farm.

\section{Summary}

In our study, a recently-developed LES framework (Wu and Porté-Agel 2011; Porté-Agel et al. 2011 ) is validated and used to investigate the interaction between boundary-layer turbulence and wind farms under neutral conditions. Two different wind-farm layouts are considered, consisting of 30 wind turbines arranged in a perfectly-aligned and a staggered configuration, respectively. The turbines occupy the same surface area of $S_{x} S_{y}=20 d^{2}$ per turbine, and are distributed in ten rows, with distance between rows of $S_{x}=5 d$, and between columns of $S_{y}=4 d$ in the aligned case. In the LES framework, the SGS stresses are parametrized using a tuning-free Lagrangian scale-dependent dynamic SGS model (Porté-Agel et al. 2000; Stoll and Porté-Agel 2006), while the turbine-induced forces (i.e., lift, drag and thrust) are modelled with two types of actuator-disk models: (a) the 'standard' actuator-disk model (ADM-NR), which calculates only the thrust force based on the one-dimensional momentum theory, and distributes it uniformly over the rotor area; and (b) the actuator-disk model with rotation (ADM-R), which uses BEM theory to calculate the lift and drag forces (that produce both thrust and rotation), and distributes them over the rotor disk based on the local blade and flow characteristics (Wu and Porté-Agel 2011; Porté-Agel et al. 2011). It should be noted that, for the ADM-NR, in this study an optimal value of the rotor thrust coefficient has been computed for each turbine based on BEM theory.

The LES results obtained with the ADM-NR and ADM-R models are compared with highresolution velocity measurements collected with hot-wire anemometry inside and above the aligned wind farm in an ABL wind tunnel (Chamorro and Porté-Agel 2011). Overall, the ADM-R yields improved predictions compared with the ADM-NR in the wake regions, where the latter model tends to underestimate the velocity deficit and the enhancement of turbulence intensity in the wakes. Therefore, including turbine-induced flow rotation and accounting for the non-uniformity of the turbine-induced forces in the ADM-R appears to be important to improve LES accuracy. Resolution sensitivity analysis shows that the simulation results obtained with the ADM-R have very little sensitivity to grid resolution as long as at least seven grid points are used across the rotor diameter in the vertical direction, and five points in the spanwise direction. The Lagrangian scale-dependent dynamic model is able to dynamically (without any parameter tuning) capture both the spatial variability as well as the scale dependence of the Smagorinsky coefficient using information of the smallest resolved scales. As expected, the value of the model coefficient $C_{S}$ decreases near the ground and in the wake shear layers in order to account for the reduced characteristic scales of the turbulence and the increased flow anisotropy.

Comparison of the simulation results for the aligned and staggered wind-farm cases shows a strong effect of wind-farm layout on the turbulent flow structure inside and above wind farms. In particular, the cumulative wakes are found to have little lateral interaction (with no interaction before the eighth row of wind turbines) in the case of the aligned wind farm. In contrast, the lateral interaction between the wakes is much stronger and happens throughout 
most of the wind farm in the case of the staggered wind farm. As a result, the growth of the cumulative wake from the staggered farm resembles more a classical internal boundary layer compared with that from the aligned farm. Overall, for finite-size wind farms such as those considered here, the staggered configuration is more efficient in extracting momentum from the flow. This is due to the fact that the distance between consecutive wind turbines is larger compared with the aligned case, which allows the wake to recover more, thus reaching a higher velocity, and the turbulence level to decrease more (leading to smaller fatigue loads) before it reaches the next downwind turbine. These differences in flow structure are also reflected in the averaged (in time and over horizontal planes) velocity profiles above the wind farms. In particular, the effective aerodynamic roughness length, an important parameter used to account for the effect of wind farms in large-scale atmospheric models, is found to be double in the case of the staggered wind farm.

Future work should extend the implementation and validation of the ADM-R model to predict turbine power production in operational wind farms, and to simulate wind-turbine wakes in a variety of cases involving different wind directions, atmospheric stability conditions, land-surface characteristics (land cover and topography) and wind-farm layouts.

Acknowledgements This research was supported by the Swiss National Science Foundation (Grant 200021 _132122), and the National Science Foundation (Grants EAR-0537856 and ATM-0854766). Computing resources were provided by the Minnesota Supercomputing Institute, and the Swiss National Supercomputing Centre under project ID s306.

\section{References}

Albertson JD, Parlange MB (1999) Surfaces length scales and shear stress: implications for land-atmosphere interactions over complex terrain. Water Resour Res 35:2121-2132

Alinot C, Masson C (2002) Aerodynamic simulations of wind turbines operating in atmospheric boundary layer with various thermal stratifications. A collection of the 2002 ASME wind energy symposium technical papers, pp 206-215

Ammara I, Leclerc C, Masson C (2002) A viscous three-dimensional differential/actuator-disk method for the aerodynamic analysis of wind farms. J Sol Energy Eng 124:345-356

Barthelmie RJ, Rathmann O, Frandsen ST, Hansen K, Politis E, Prospathopoulos J, Chaviaropoulos PK, Rados K, Cabezón D, Schlez W, Phillips J, Neubert A, Schepers J, van der Pijl S (2007) Modelling and measurements of wakes in large wind farms. J Phys Conf Ser 75:012049

Barthelmie RJ, Hansen K, Frandsen ST, Rathmann O, Schepers JG, Schlez W, Phillips J, Rados K, Zervos A, Politis ES, Chaviaropoulos PK (2009) Modelling and measuring flow and wind turbine wakes in large wind farms offshore. Wind Energy 12:431-444

Betz A (1926) Windenergie und Ihre Ausnutzung durch Windmüllen

Calaf M, Meneveau C, Meyers J (2010) Large eddy simulation study of fully developed wind-turbine array boundary layers. Phys Fluids 22:015110

Chamorro L, Porté-Agel F (2009) A wind-tunnel investigation of wind-turbine wakes: boundary-layer turbulence effects. Boundary-Layer Meteorol 132:129-149

Chamorro LP, Porté-Agel F (2011) Turbulent flow inside and above a wind farm: a wind-tunnel study. Energies 4:1916-1936

Frandsen S (1992) On the wind-speed reduction in the center of large clusters of wind turbines. J Wind Eng Ind Aerodyn 39:251-265

Gómez-Elvira R, Crespo A, Migoya E, Manuel F, Hernández J (2005) Anisotropy of turbulence in wind turbine wakes. J Wind Eng Ind Aerodyn 93:797-814

Hansen M, Sørensen JN, Voutsinas S, Sørensen N, Madsen HA (2006) State of the art in wind turbine aerodynamics and aeroelasticity. Prog Aerosp Sci 42:285-330

Hansen KS, Barthelmie RJ, Jensen LE, Sommer A (2012) The impact of turbulence intensity and atmospheric stability on power deficits due to wind turbine wakes at horns rev wind farm. Wind Energy 15:183-196

International Standard IEC 61400-1 (2005) Wind turbines-part 1: design requirements, appendix D: wake and wind farm turbulence, 3rd edn. International Electrotechnical Commission 
Jimenez A, Crespo A, Migoya E, Garcia J (2007) Advances in large-eddy simulation of a wind turbine wake. J Phys Conf Ser 75:012041

Jimenez A, Crespo A, Migoya E, Garcia J (2008) Large-eddy simulation of spectral coherence in a wind turbine wake. Environ Res Lett 3:015004

Jimenez A, Crespo A, Migoya E, Garcia J (2010) Application of a les technique to characterize the wake deflection of a wind turbine in yaw. Wind Energy 13:559-572

Kasmi AE, Masson C (2008) An extended $\kappa-\varepsilon$ model for turbulent flow through horizontal-axis wind turbines. J Wind Eng Ind Aerodyn 96:103-122

Kleissl J, Meneveau C, Parlange MB (2003) On the magnitude and variability of subgrid-scale eddy-diffusion coefficients in the atmospheric surface layer. J Atmos Sci 60:2372-2388

Lettau H (1969) Note on aerodynamic roughness-parameter estimation on the basis of roughness-element description. J Appl Meteorol 8:828-832

Lu H, Porté-Agel F (2011) Large-eddy simulation of a very large wind farm in a stable atmospheric boundary layer. Phys Fluids 23:065101

Markfort CD, Zhang W, Porté-Agel F (2012) Turbulent flow and scalar transport through and over aligned and staggered wind farms. J Turbul. doi:10.1080/14685248.2012.709635

Masson C, Ammara I, Paraschivoiu I (1997) An aerodynamic method for the analysis of isolated horizontalaxis wind turbines. Int J Rotating Mach 3:21-32

Phillips AB, Turnock SR, Furlong M (2009) Evaluation of manoeuvring coefficients of a self-propelled ship using a blade element momentum propeller model coupled to a Reynolds averaged Navier Stokes flow solver. Ocean Eng 36:1217-1225

Porté-Agel F (2004) A scale-dependent dynamic model for scalar transport in large-eddy simulations of the atmospheric boundary layer. Boundary-Layer Meteorol 112:81-105

Porté-Agel F, Meneveau C, Parlange MB (2000) A scale-dependent dynamic model for large-eddy simulations: application to a neutral atmospheric boundary layer. J Fluid Mech 415:261-284

Porté-Agel F, Meneveau C, Parlange MB, Eichinger WE (2001) A priori field study of the subgrid-scale heat fluxes and dissipation in the atmospheric surface layer. J Atmos Sci 58:2673-2698

Porté-Agel F, Pahlow M, Meneveau C, Parlange MB (2001) Atmospheric stability effect on subgrid-scale physics for large-eddy simulation. Adv Water Resour 24:1085-1102

Porté-Agel F, Wu YT, Lu H, Conzemius RJ (2011) Large-eddy simulation of atmospheric boundary layer flow through wind turbines and wind farms. J Wind Eng Ind Aerodyn 99:154-168

Schetz JA, Fuhs AE (1996) Handbook of fluid dynamics and fluid machinery. Wiley, New York, 2776 pp

Smagorinsky J (1963) General circulation experiments with the primitive equations: I. The basic experiment. Mon Weather Rev 91:99-164

Sørensen JN (2011) Aerodynamic aspects of wind energy conversion. Annu Rev Fluid Mech 43:427-448

Sørensen JN, Kock CW (1995) A model for unsteady rotor aerodynamics. J Wind Eng Ind Aerodyn 58:259_ 275

Sørensen JN, Myken A (1992) Unsteady actuator disc model for horizontal axis wind turbines. J Wind Eng Ind Aerodyn 39:139-149

Sørensen JN, Shen WZ (2002) Numerical modeling of wind turbine wakes. J Fluids Eng 124:393-399

Stoll R, Porté-Agel F (2006) Dynamic subgrid-scale models for momentum and scalar fluxes in large-eddy simulations of neutrally stratified atmospheric boundary layers over heterogeneous terrain. Water Resour Res 42:W01409

Stoll R, Porté-Agel F (2008) Large-eddy simulation of the stable atmospheric boundary layer using dynamic models with different averaging schemes. Boundary-Layer Meteorol 126:1-28

Sunada S, Sakaguchi A, Kawachi K (1997) Airfoil section characteristics at a low Reynolds number. J Fluids Eng 119:129-135

Tseng YH, Meneveau C, Parlange MB (2006) Modeling flow around bluff bodies and predicting urban dispersion using large eddy simulation. Environ Sci Technol 40:2653-2662

Vermeer LJ, Sørensen JN, Crespo A (2003) Wind turbine wake aerodynamics. Prog Aerosp Sci 39:467-510

Wan F, Porté-Agel F (2011) Large-eddy simulation of stably-stratified flow over a steep hill. Boundary-Layer Meteorol 138:367-384

Wan F, Porté-Agel F, Stoll R (2007) Evaluation of dynamic subgrid-scale models in large-eddy simulations of neutral turbulent flow over a two-dimensional sinusoidal hill. Atmos Environ 41:2719-2728

Wu YT, Porté-Agel F (2011) Large-eddy simulation of wind-turbine wakes: evaluation of turbine parametrisations. Boundary-Layer Meteorol 138:345-366

Zhang W, Markfort CD, Porté-Agel F (2012) Near-wake flow structure downwind of a wind turbine in a turbulent boundary layer. Exp Fluids 52:1219-1235 